\title{
Interleukin-7 restores lymphocytes in septic shock: the IRIS-7 randomized clinical trial
}

Bruno Francois, ${ }^{1,2,3}$ Robin Jeannet, ${ }^{2}$ Thomas Daix, ${ }^{1,2}$ Andrew H. Walton, ${ }^{4}$ Matthew S. Shotwell, ${ }^{5}$ Jacqueline Unsinger, ${ }^{4}$ Guillaume Monneret, ${ }^{6,7}$ Thomas Rimmelé, ${ }^{7,8}$ Teresa Blood, ${ }^{4}$ Michel Morre, ${ }^{9}$ Anne Gregoire, ${ }^{9}$ Gail A. Mayo, ${ }^{10}$ Jane Blood, ${ }^{4}$ Scott K. Durum, ${ }^{11}$ Edward R. Sherwood, ${ }^{10,12}$ and Richard S. Hotchkiss $s^{4,13,14}$

IIntensive Care Unit, and 'Inserm CIC-1435, Dupuytren University Hospital, Limoges, France. ${ }^{3}$ Inserm UMR-1092, University of Limoges, Limoges, France. ${ }^{4}$ Department of Anesthesiology, Washington University School of Medicine, St. Louis, Missouri, USA. ${ }^{5}$ Department of Biostatistics, Vanderbilt University, Nashville, Tennessee, USA. ${ }^{6}$ Cellular Immunology Laboratory, Edouard Herriot Hospital, Hospices Civils de Lyon, Lyon, France. ${ }^{7}$ EA 7426 PI3 “Pathophysiology of Injury-induced Immunosuppression", Université Claude Bernard Lyon I-Biomérieux-Hospices Civils de Lyon, Lyon, France. ${ }^{8}$ Anesthesiology and Intensive Care Medicine, Edouard Herriot Hospital, Hospices Civils de Lyon, Lyon, France. ${ }^{9}$ Revimmune SAS, Paris, France. ${ }^{10}$ Department of Anesthesiology, Vanderbilt University Medical Center, Nashville, Tennessee, USA. "Cytokines and Immunity Section, National Cancer Institute, Bethesda, Maryland, USA. ${ }^{2}$ Department of Pathology, Microbiology and Immunology, Vanderbilt University Medical Center, Nashville, Tennessee, USA. ${ }^{13}$ Department of Medicine, and ${ }^{14}$ Department of Surgery, Washington University School of Medicine, St. Louis, Missouri, USA.

BACKGROUND. A defining pathophysiologic feature of sepsis is profound apoptosis-induced death and depletion of $\mathrm{CD4}^{+}$and $\mathrm{CD}^{+} \mathrm{T}$ cells. Interleukin-7 (IL-7) is an antiapoptotic common $\gamma$-chain cytokine that is essential for lymphocyte proliferation and survival. Clinical trials of IL-7 in over 390 oncologic and lymphopenic patients showed that IL-7 was safe, invariably increased CD4+ and CD8 ${ }^{+}$ lymphocyte counts, and improved immunity.

METHODS. We conducted a prospective, randomized, double-blind, placebo-controlled trial of recombinant human IL-7 (CYT107) in patients with septic shock and severe lymphopenia. Twentyseven patients at academic sites in France and the United States received CYT107 or placebo for 4 weeks. Primary aims were to determine the safety of CYT107 in sepsis and its ability to reverse lymphopenia.

RESULTS. CYT107 was well tolerated without evidence of inducing cytokine storm or worsening inflammation or organ dysfunction. CYT107 caused a 3- to 4-fold increase in absolute lymphocyte counts and in circulating $\mathrm{CD}^{+}$and $\mathrm{CD8} 8^{+} \mathrm{T}$ cells that persisted for weeks after drug administration. CYT107 also increased T cell proliferation and activation.

CONCLUSIONS. This is the first trial of an immunoadjuvant therapy targeting defects in adaptive immunity in patients with sepsis. CYT107 reversed the marked loss of CD4+ and CD8+ immune effector cells, a hallmark of sepsis and a likely key mechanism in its morbidity and mortality. CYT107 represents a potential new way forward in the treatment of patients with sepsis by restoring adaptive immunity. Such immune-based therapy should be broadly protective against diverse pathogens including multidrug resistant bacteria that preferentially target patients with impaired immunity.

TRIAL REGISTRATION. Trials registered at clinicaltrials.gov: NCT02640807 and NCT02797431.

Conflict of interest: M. Morre and A. Gregoire are Revimmune employees.

Submitted: November 30, 2017 Accepted: January 25, 2018 Published: March 8, 2018

Reference information: JCI Insight. 2018;3(5):e98960. https://doi.org/10.1172/jci. insight. 98960.
FUNDING. Revimmune, NIH National Institute of General Medical Sciences GM44118.

\section{Introduction}

A defining feature of sepsis is a profound apoptosis-induced depletion of lymphocytes (1-4). Postmortem studies of patients who died of sepsis demonstrate extensive loss of lymphocytes in spleens, intestines, and other organs $(1,4)$. The tissue lymphocyte depletion occurring during sepsis is accompanied by a decrease 


\section{Trial Enrollment}

Enrollment

All ICUs were screened Monday through Friday for eligible patients Approached for enrollment in study $(n=34)$

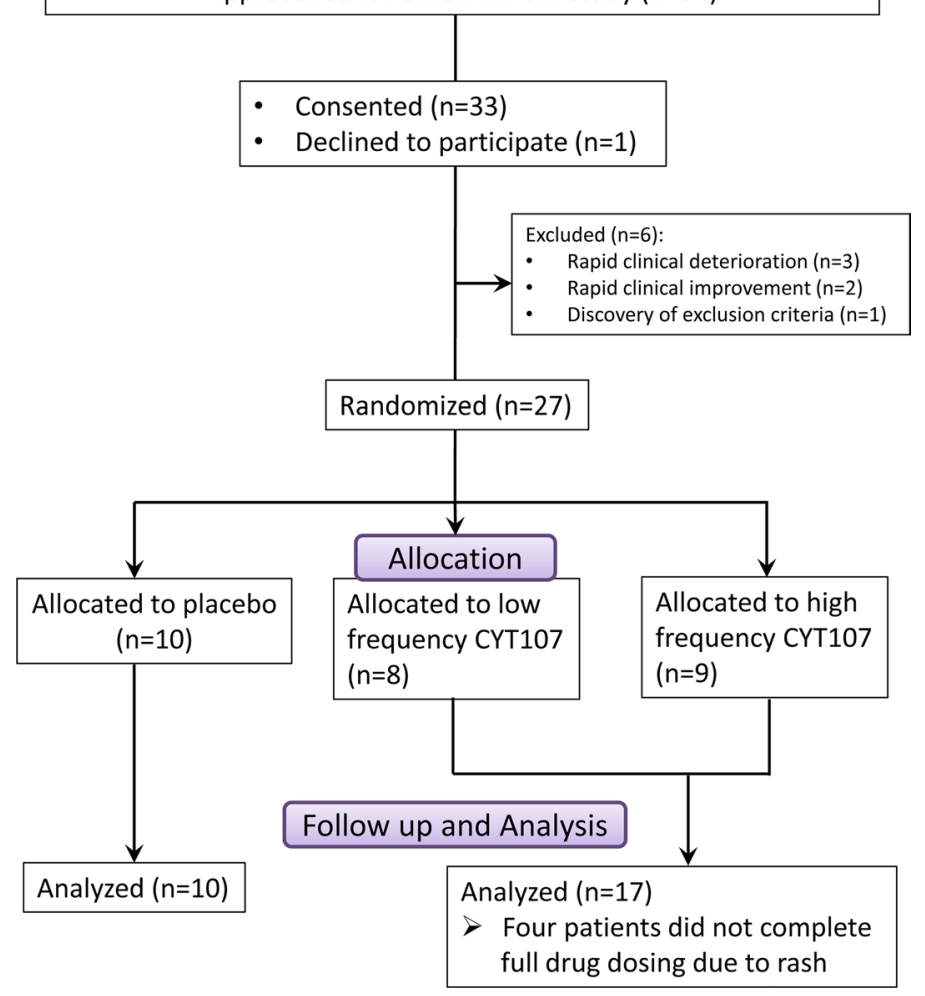

Figure 1. Trial enrollment details. Details on patient enrollment, randomization, allocation, and follow-up and analysis are presented. Medical, Surgical, Neurosurgical, and Cardiothoracic Intensive Care Units were screened daily Monday through Friday for patients on antibiotics and vasopressors. After identifying patients in presumed septic shock, patients were further screened for inclusion and exclusion criteria as per the protocol.

in the absolute number of circulating lymphocytes, i.e., lymphopenia (5-8). This lymphopenia that is present in sepsis consists of the loss of many different classes of lymphocytes including $\mathrm{CD} 4^{+}$ and $\mathrm{CD}^{+} \mathrm{T}$ cells, B cells, and natural killer cells $(1,9) . \mathrm{CD}^{+} \mathrm{T}$ cell counts in patients with sepsis are often as low as that occurring in patients with life-threatening AIDS (10). Lymphocytes are essential for an effective host defense against invading pathogens (11). Studies in patients who have undergone bone marrow or solid organ transplantation show a direct correlation between low $\mathrm{CD}^{+}$cell counts and the incidence of new onset opportunistic infections and death $(12,13)$. Similarly, numerous studies have shown that septic patients with more severe and persistent lymphopenia have a higher mortality compared with septic patients who are either not lymphopenic or who have a lesser degree of lymphopenia (5-8).

A key question is whether the lymphocyte depletion in sepsis is merely an epiphenomenon or whether it plays a central role in lethality in sepsis. Several mechanistic studies have addressed this critical issue. Studies by multiple independent laboratories showed that mice whose lymphocytes were resistant to sepsisinduced apoptosis because of either overexpression of the antiapoptotic protein $\mathrm{Bcl}-2$ or deletion of the proapoptotic protein

Bim have improved survival in sepsis (14-19). Furthermore, studies also demonstrate that adoptive transfer of lymphocytes that are resistant to sepsis-induced apoptosis, but not adoptive transfer of normal apoptosis-susceptible lymphocytes, improves sepsis survival (15). Collectively, these animal studies together with patient studies showing a close correlation between the degree of lymphopenia and survival strongly suggest that the extensive lymphocyte depletion is fundamental to the pathophysiology of sepsis $(20,21)$.

Interleukin-7 (IL-7) is a potent Bcl-2-inducing, antiapoptotic, common $\gamma$-chain cytokine that is required for lymphocyte survival and expansion (22-24). In addition to its antiapoptotic properties, IL-7 induces robust proliferation of $\mathrm{CD}^{+}$and $\mathrm{CD} 8^{+} \mathrm{T}$ cells. Given its ability to prevent lymphocyte apoptosis, induce lymphocyte proliferation, and improve lymphocyte function, IL-7 has been tested by several groups in animal models of sepsis (25-27). These studies showed that IL-7 prevented lymphocyte apoptosis, restored $\mathrm{CD}^{+}$and $\mathrm{CD}^{+} \mathrm{T}$ cell function, and improved survival in animal models of bacterial and fungal sepsis (25-27). Importantly, IL-7 was also effective in preventing lymphocyte apoptosis, improving T cell cytokine production, and restoring lymphocyte metabolism in ex vivo studies conducted on peripheral blood from patients with septic shock $(28,29)$.

Although not currently approved for clinical use, CYT107, a glycosylated recombinant human IL-7, has been administered in clinical trials to over 390 adult and pediatric patients for a variety of disorders including HIV, idiopathic lymphopenia, cancer, hepatitis C, and bone marrow reconstitution following stem cell transplantation (30-35). In clinical trials of HIV-infected patients who had lymphopenia despite antiretroviral therapy, CYT107 caused a 2- to 4-fold dose-dependent increase in circulating CD4 ${ }^{+}$and $\mathrm{CD}^{+} \mathrm{T}$ cells (31). CYT107 consistently increased circulating lymphocyte counts and was well tolerated, with rash being the only serious adverse effect (30-35). Recently, CYT107 was administered on a compassionate basis to patients with lymphopenia and John Cunningham (JC) virus-induced progressive multifocal leukoencephalopathy $(36,37)$. In this desperate setting, CYT107 typically increased lymphocyte counts, decreased JC viral load, and led to resolution of neurologic symptoms $(36,37)$. 
A

\begin{tabular}{|c|c|c|c|c|c|c|c|c|c|}
\hline \multirow{3}{*}{$\begin{array}{l}\text { High } \\
n=9 \\
\text { Low } \\
n=8\end{array}$} & \multicolumn{2}{|c|}{$\begin{array}{l}\text { AL-7 } \\
\text { Placebo }\end{array}$} & \multicolumn{4}{|c|}{ Days } & & & \\
\hline & 0 & $\Delta$ & 7 & $\Delta$ & $14 \Delta$ & $\Delta$ & 214 & $\boldsymbol{i}$ & 28 \\
\hline & $0<$ & $\Delta$ & 7 & $\Delta$ & $14^{-1}$ & $\boldsymbol{\Delta}$ & 214 & $\boldsymbol{\Delta}$ & 28 \\
\hline-10 & $0 \Delta$ & $\Delta$ & $7 \Delta$ & $\Delta$ & $14 \Delta$ & $\bar{\Delta}$ & $21 \Delta$ & $\boldsymbol{\Delta}$ & 28 \\
\hline
\end{tabular}
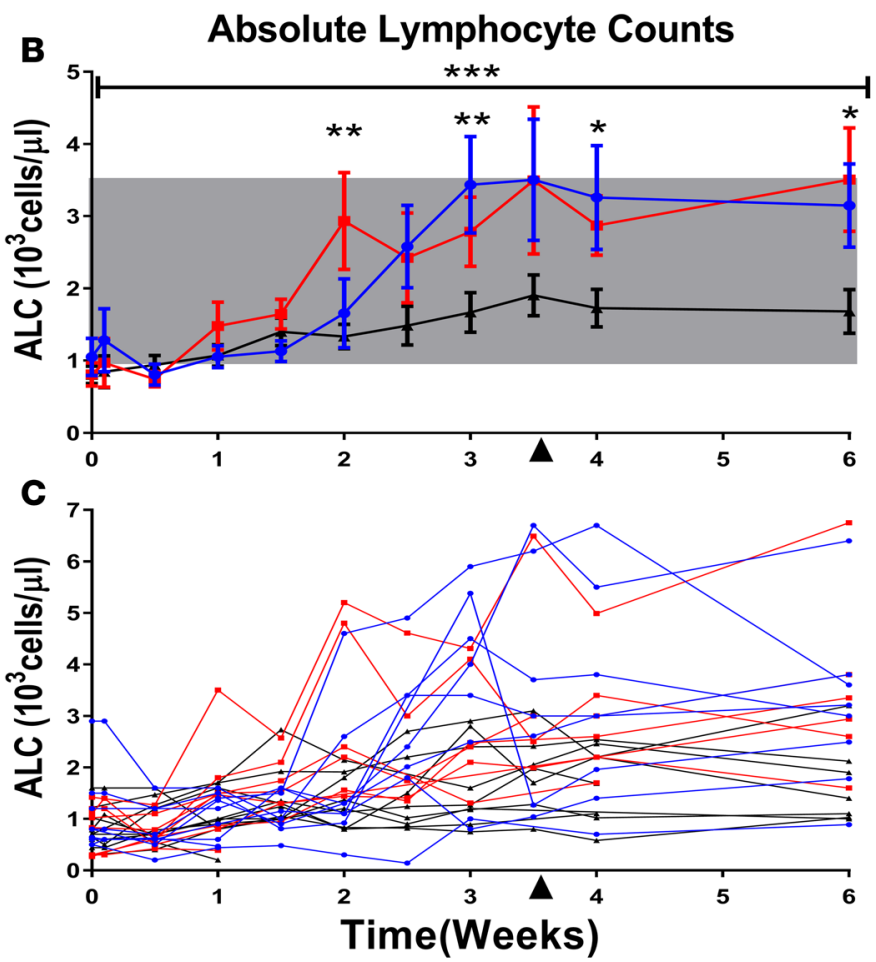

× Placebo $(n=10) \rightarrow-\operatorname{CYT107} \operatorname{Low}(n=8) \rightarrow \operatorname{CYT107} \operatorname{High}(n=9)$
Figure 2. CYT107 increases absolute lymphocyte counts. (A) Patients were treated with $10 \mu \mathrm{g} / \mathrm{kg}$ CYT107, a recombinant human interleukin-7 (IL-7) using 2 different dosing regimens. Both CYT107treated groups received CYT107 twice a week for the first week. Thereafter, one group was treated with CYT107 once a week (low frequency) while the second CYT107 treated group was treated with CYT107 twice a week (high frequency). Therapy with CYT107 was continued for a total of 4 weeks or until the patient was discharged from the hospital. The maximum number of doses of CYT107 that a patient could receive was 5 or 8 doses respectively for the low- and high-dose regimens of CYT107. All patients received injections twice a week. (B) The CYT107 treatment effect on average absolute lymphocyte counts (ALCs) was significant for both high- and low-frequency treatment regimens. The gray-shaded region represents the upper and lower limit of normal for ALCs. The CYT107 low-frequency group (red color) was greater than the placebo-treated group at days 15 and 42, and the CYT107 high-frequency group (blue color) was greater than placebo-treated at days 22 and 29 . ${ }^{*} P<0.05$, ${ }^{*} P<0.01,{ }^{* *} P<0.001$. Statistical tests were conducted using a Wald-type multiple-degree-of-freedom method. Dark arrowhead represents the last day of treatment. Values reported are mean \pm SEM. $n=10,8$, and 9 for placebo, low-frequency CYT107-treated, and high-frequency CYT107-treated patients, respectively. (C) The CYT107 treatment effect on ALCs is displayed for each patient over the study duration.

We conducted a prospective, multicenter, randomized, double-blind, placebo-controlled phase IIb trial of CYT107 in patients with septic shock and severe lymphopenia of 900 lymphocytes/ $\mu$ l or lower. Clinical immunoadjuvant trials to date have targeted innate immunity. Importantly, this study represents the first immunotherapeutic trial to our knowledge focused on augmenting the adaptive immune system in patients with sepsis. The primary purpose of the trial was to assess the safety and tolerability of CYT107 in this critically ill patient population with sepsis. The second goal of the trial was to determine if CYT107 could reverse the profound sepsis-induced lymphopenia and improve the absolute numbers of circulating $\mathrm{CD}^{+}$and $\mathrm{CD}^{+} \mathrm{T}$ cells. Additional aims of the study were to examine the effect of CYT107 on markers of lymphocyte proliferation and activation, and incidence of secondary, hospital-acquired infections.

Herein we show that therapy with CYT107 in septic patients was well tolerated without any evidence of inducing a cytokine storm or worsening ongoing inflammation. CYT107 was effective in reversing the profound sepsis-induced lymphopenia and caused a 3- to 4-fold increase in the absolute lymphocyte count and in circulating $\mathrm{CD}^{+}$and $\mathrm{CD} 8^{+} \mathrm{T}$ cells. Thus, CYT107 reversed a key pathogenic hallmark of sepsis that likely plays a major role in sepsis-associated morbidity and mortality. Given CYT107's extensive safety record in critically ill patients, its ability to improve survival in multiple animal models of sepsis, and its documented efficacy in patients with life-threatening viral infections, additional clinical trials of CYT107 in sepsis should be conducted. Targeting the dysfunctional adaptive immune system in sepsis with IL-7 may represent a new way forward against this highly lethal disease.

\section{Results}

See supplemental materials and methods for additional details. 
Table 1. Clinical characteristics

\begin{tabular}{|c|c|c|c|}
\hline & Placebo $(n=10)$ & IL-7 $(n=17)$ & $P$ value \\
\hline Age, mean (range) & $56(33-77)$ & $65(33-82)$ & 0.197 \\
\hline Gender, M/F & $8 / 2$ & $13 / 4$ & 0.831 \\
\hline Baseline SOFA score, mean (range) & $9(3-16)$ & $7(2-12)$ & 0.194 \\
\hline Baseline APACHE II score, mean (range) & $17(3-30)$ & $14(8-23)$ & 0.461 \\
\hline Infections, number: & & & 0.947 \\
\hline Abdominal infection & 3 & 6 & \\
\hline Mediastinitis & 1 & 1 & \\
\hline Necrotizing fasciitis & 1 & 2 & \\
\hline Pneumonia & 3 & 6 & \\
\hline Urinary tract infection & 1 & 1 & \\
\hline Days in ICU, median (range) & $19(7-43)$ & $16(4-70)$ & 0.939 \\
\hline
\end{tabular}

SOFA, sequential organ failure assessment; APACHE II, acute physiologic assessment and chronic health evaluation II.

\section{Study design and participants}

This double-blind, randomized, placebo-controlled phase IIb trial of CYT107 was conducted at 4 academic sites, with 2 sites in the United States (Vanderbilt University School of Medicine and Washington University School of Medicine) and 2 sites in France (University Hospital of Limoges and Edouard Herriot Hospital). Following FDA approval of the study, the Institutional Review Boards at Vanderbilt University and Washington University approved the protocols. For France, the study protocol was approved by the Committee for the Protection of Persons (CPP) and the National Agency for Drug Safety (ANSM). All patients or their legal representatives gave written informed consent prior to inclusion in the study. Both the US and French teams used identical protocols registered on Clinicaltrials.gov, NCT02640807 and NCT02797431 respectively, for the 2 countries. The data from all 4 sites were combined for analysis.

Inclusion criteria. Twenty-seven patients who met the criteria for vasopressor-dependent septic shock with at least one organ failure defined by a sequential organ failure assessment (SOFA) score of 2 or higher persisting at 48-120 hours after intensive care unit (ICU) admission were enrolled into the study (Figure 1) (38). See supplemental materials for further patient enrollment details and additional inclusion and exclusion criteria. An additional entry criterion for inclusion was lymphopenia with an absolute lymphocyte count of 900 lymphocytes/ $\mu 1$ or lower obtained within $24-48$ hours prior to providing consent. Lymphopenia was selected as an inclusion criterion because persistent lymphopenia identifies patients with immunosuppression and a higher mortality (5-8).

Exclusion criteria. Patients were excluded for evidence of autoimmune disorders, active hematological diseases, cancer with current chemo- or radiotherapy, treatment with corticosteroids equivalent to a dose of $300 \mathrm{mg} /$ day or higher of hydrocortisone, and treatment with immunosuppressive medications.

CYT107 dosing regimens. Patients received either CYT107 (glycosylated recombinant human IL-7) or placebo via the intramuscular route. There were 2 different dosing regimens for CYT107; both groups received $10 \mu \mathrm{g} / \mathrm{kg}$ CYT107 twice a week for the first week (Figure 2A). Thereafter, one group was treated with $10 \mu \mathrm{g} /$ $\mathrm{kg}$ CYT107 once a week (low frequency), while the second CYT107-treated group was treated with CYT107 $10 \mu \mathrm{g} / \mathrm{kg}$ twice a week (high frequency). Therapy with CYT107 was continued for a total of 4 weeks (last dose administered on day 25 after trial initiation) or until the patient was discharged from the hospital. The maximum number of doses of CYT107 that a patient could receive was 5 or 8 doses respectively for the lowand high-dose regimens of CYT107. All patients were injected twice a week with either placebo or CYT107 to maintain blinding of investigators and treating physicians (see Figure 2A for protocol).

Patient characteristics. There were no significant differences in the patient age, gender, site of infection, ICU days, SOFA, or acute physiologic assessment and chronic health evaluation (APACHE) II scores (Table 1). The most common sites of infection were pneumonia and abdominal infection (peritonitis). Pneumonia was present in 6 of $17(35 \%)$ CYT107-treated patients and 3 of $10(30 \%)$ of placebo-treated patients. Peritonitis was present in 6 of 17 (35\%) of CYT107-treated patients and 3 of 10 (30\%) placebo-treated patients. 


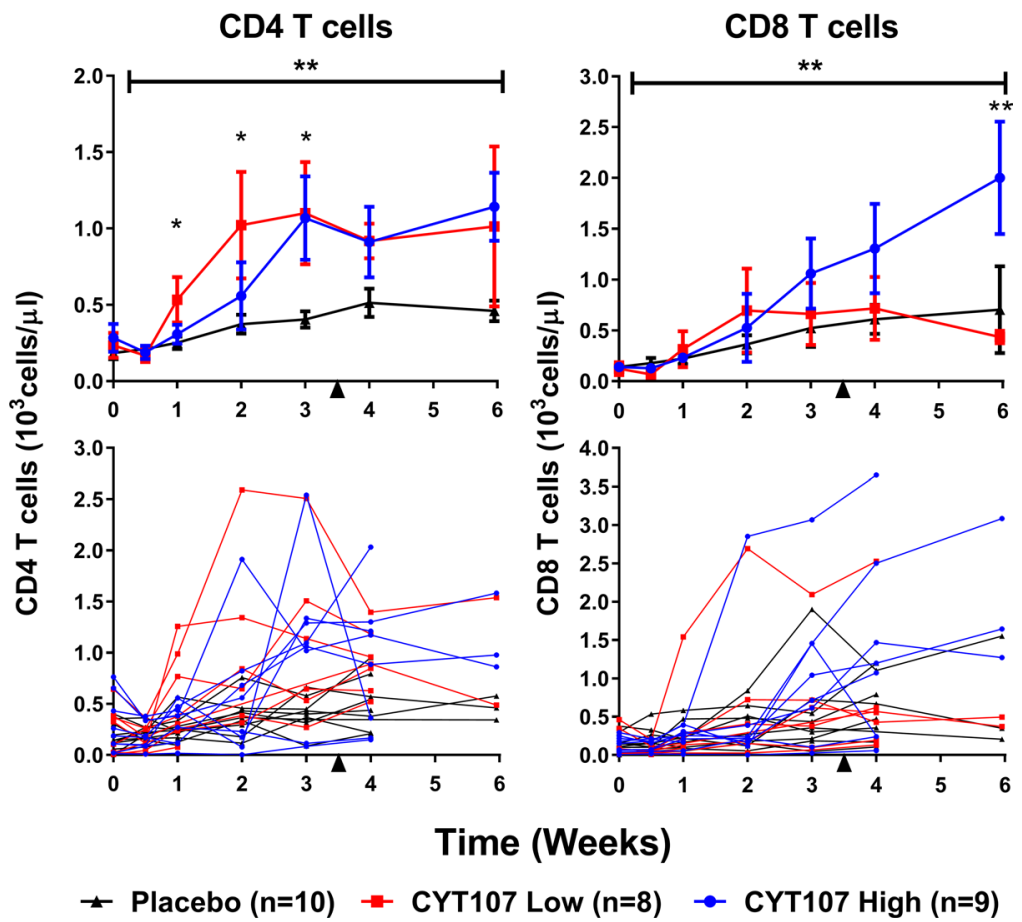

Figure 3. CYT107 increased $\mathrm{CD}^{+}$and $\mathrm{CDB}^{+} \mathrm{T}$ cell counts. (A) The effect of CYT107 on the averaged CD4+ and CD8 ${ }^{+} \mathrm{T}$ cell counts, considered for study duration, was significant for both $\mathrm{CD}^{+}$and $\mathrm{CD} 8^{+} \mathrm{T}$ cells. The low-frequency regimen (red color) significantly increased CD4+ and the high-frequency regimen (blue color) increased $\mathrm{CD} 8^{+} \mathrm{T}$ cells compared with placebo. Low-frequency CYT107 therapy increased CD4 ${ }^{+} \mathrm{T}$ cells at days 8,15 , and 22 . High-frequency CYT107 therapy increased $\mathrm{CD} 8{ }^{+} \mathrm{T}$ cells at day $42 .{ }^{*} P<0.05,{ }^{*} P<0.01$. Statistical tests were conducted using a Wald-type multipledegree-of-freedom method. Values reported are mean \pm SEM. $n=10,8$, and 9 for placebo, low-frequency CYT107, and high-frequency CYT107-treated patients, respectively. (B) The effect of CYT107 on individual patient $\mathrm{CD}^{+}$and CD8 ${ }^{+}$ $T$ cell counts is presented. Note that the patients at US sites had quantification of CD4 ${ }^{+}$and $C D 8^{+} T$ cell counts for 6 weeks, while the patients at French sites had quantification out to 4 weeks only.

\section{Safety and tolerability of CYT107}

Of the 27 patients who were randomized into the study, 9 patients were randomized to the high-frequency regimen, 8 patients were randomized to the low-frequency CYT107 regimen, and 10 patients were randomized into placebo. The mean number of doses of CYT107 that the patients in the low- and high-frequency protocols received was $4.3 \pm 0.3$ and $6.1 \pm 0.8$, respectively. CYT107 was well tolerated with no evidence of induction of cytokine storm or excessive inflammation. Specifically, administration of CYT107 was not temporally associated with new onset or worsening of existing fever, tachycardia, hypotension, or evidence of organ injury.

Serial quantification of the effect of CYT107 on the proinflammatory cytokines TNF- $\alpha$ and IL- 6 and the antiinflammatory cytokine IL-10 was performed on patient plasma samples (Supplemental Figure 1, A-F; supplemental material available online with this article; https://doi.org/10.1172/jci.insight.98960DS1). The baseline value for TNF- $\alpha$ in patients, i.e., prior to treatment with CYT107 or placebo, was $17.5 \pm 3.1$ $\mathrm{pg} / \mathrm{ml}(n=22$ patients). Most CYT107-treated patients showed no increase in TNF- $\alpha$ above the beginning baseline values, while 2 patients had a transient increase at one time point (Supplemental Figure 1C). Baseline IL-6 levels were elevated in septic patients, i.e., $158.5 \pm 30.5 \mathrm{pg} / \mathrm{ml}(n=22$ patients). The majority of CYT107-treated patients showed no effect of drug administration on IL-6. There was a modest increase in IL-6 levels in 2 CYT107-treated patients but also in 1 placebo-treated patient (Supplemental Figure 1A). Baseline IL-10 levels were elevated in septic patients, i.e., $99.0 \pm 17.0 \mathrm{pg} / \mathrm{ml}(n=22$ patients). There was an increase in IL-10 in 2 CYT107-treated patients and in 1 placebo-treated patient (Supplemental Figure 1B).

Serial laboratory testing for serum creatinine, a measure of renal function, showed no difference in CYT107- versus placebo-treated patients (Supplemental Figure 2). Similarly, there were no differences in serial liver function tests in CYT107- versus placebo-treated patients (Supplemental Figure 3).

The only adverse effects attributable to CYT107 were injection site reactions varying from a grade 1-3 rash, as scored according to the Division of AIDS (DAIDS) version 2 toxicity scale. Most injection site reactions that occurred were small and less than 0.5 to $2.0 \mathrm{~cm}$ in diameter (grade 1). Six patients developed a grade 2-3 injection site reaction consisting of a raised red rash without vesicles or blistering involving $20 \%-70 \%$ of the surface area of one upper or lower extremity. Despite the rash, 2 patients continued to receive CYT107 for the entire 8-dose regimen. CYT107 therapy was discontinued in 4 patients for progression of the rash as follows: one patient discontinued after 3 doses, one patient discontinued after 4 doses, one patient discontinued after 6 doses, and one patient discontinued after 7 doses. Biopsies of skin rashes in 2 of the patients showed CD3-positive lymphocytic infiltration (Supplemental Figure 4). All rashes resolved without complication after completion of CYT107. 

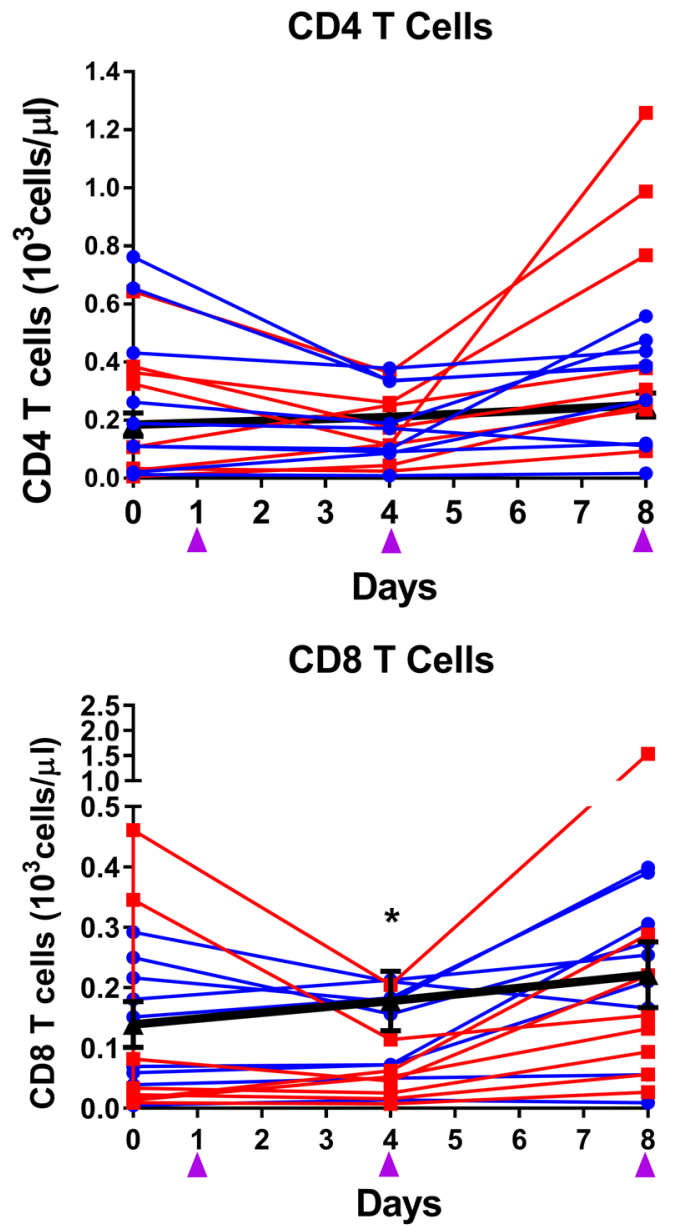

Figure 4. CYT107 causes an initial drop in circulating $\mathrm{CD8}^{+} \mathrm{T}$ cells. CYT107 caused an early transient decrease in $\mathrm{CD}^{+}$and $\mathrm{CD} 8^{+} \mathrm{T}$ cells prior to significantly increasing lymphocyte counts. Individual septic patients treated with low- and high-dose CYT107 regimens are depicted in red and blue color, respectively. The placebo-treated patients are presented as a group and depicted with a solid black line. At day $4, \mathrm{CD}^{+} \mathrm{T}$ cells were significantly decreased in patients receiving low-frequency regimen CYT107 (red color) compared with placebo $(P \leq 0.05)$. The effect of CYT107 to decrease CD4+ T cells did not quite achieve statistical significance. Statistical tests were conducted using a Wald-type multiple-degree-of-freedom method. Values reported are mean \pm SEM. $n=10,8$, and 9 for placebo, low-frequency CYT107, and high-frequency CYT107-treated patients, respectively.

\footnotetext{
\pm Placebo $(n=10)-$ CYT107 Low $(n=8)-0$ CYT107 High $(n=9)$

$\Delta$ Injection Days
}

CYT107 increased the absolute lymphocyte count

The major biological endpoint of the trial was to determine if CYT107 increases the absolute lymphocyte count in patients with sepsis. Both low- and high-frequency treatment groups significantly increased the absolute lymphocyte count over the course of the study (combined treatment effect: $P<0.001$ ) (Figure 2, B and C). At day 29, shortly after the end of therapy, both the low- and high-frequency CYT107-treated patient groups had a marked and comparable 3.5- to 4.5-fold average increase in their absolute lymphocyte counts compared with their initial values (Figure 2B). Specifically, at day 29, the absolute lymphocyte counts were $2.98 \pm 0.47 \times 10^{3}$ and $3.26 \pm 0.72 \times 10^{3}$ lymphocytes/ $\mu 1$ for the low- and high-frequency CYT107-treated groups, respectively. After baseline adjustment, at day 29 the absolute lymphocyte count among patients in the low- and high-frequency CYT107-treated groups was greater by $0.99 \times 10^{3}(95 \%$ CI: $0.14,2.13)$ and $1.30 \times 10^{3}$ lymphocytes $/ \mu 1$ (95\% CI: $\left.0.20,2.40\right)$, relative to the placebo-treated group (combined treatment effect at day 29: $P=0.004$ ) (Supplemental Figure 5). Note that there was a smaller gradual increase in the absolute lymphocyte count in the placebo-treated group by the end of 4 weeks (Figure $2 \mathrm{~B}$ ). In this regard, patients who survive sepsis typically have a slow return of their absolute lymphocyte count towards low-normal values while the absolute lymphocyte count usually remains low in patients who ultimately die of sepsis (6). The increase in the absolute lymphocyte count in both CYT107-treated groups approximated the upper limit of normal for most hospital laboratories, i.e., $3.5 \times 10^{3}$ lymphocytes $/ \mu 1$.

The increase in absolute lymphocyte count induced by CYT107 persists after therapy Examination of the trajectory for the patient absolute lymphocyte counts showed that the ability of CYT107 to increase circulating lymphocytes persisted for at least 2-4 weeks following cessation of CYT107 therapy 
A Absolute Monocyte Counts

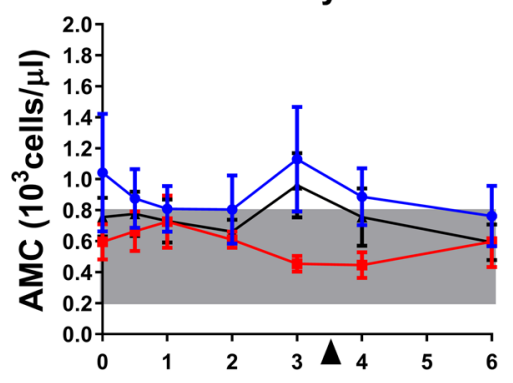

B

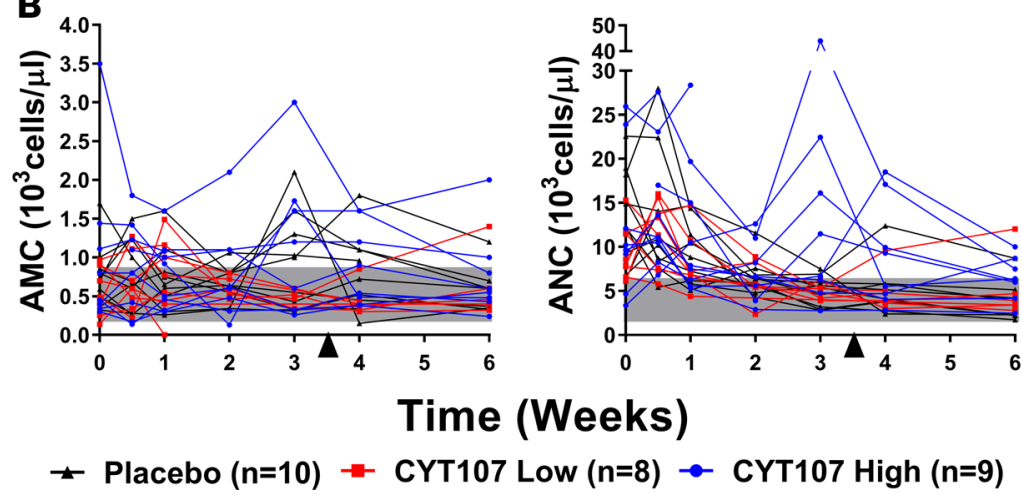

Figure 5. CYT107 effect on absolute neutrophil and absolute monocyte counts. (A) The top right panel depicts the patients' absolute neutrophil counts (ANCS) with the shaded gray area showing the normal counts for healthy individuals. Septic patients had an increase in their ANCs that returned to the normal range as their sepsis improved. There was an overall treatment effect of CYT107 on ANC. Further, there was an effect of high-frequency CYT107 to increase ANCs at weeks 3, 4, and 6. The top left panel depicts patients' absolute monocyte counts (AMCs). Patients' AMCs were generally within the normal range (shaded gray region) during their sepsis and not different in the 3 treatment groups. Statistical tests were conducted using a Wald-type multiple-degree-of-freedom method. Values reported are mean \pm SEM. $n=10,8$, and 9 for placebo, low-frequency CYT107, and high-frequency CYT107-treated patients, respectively. ${ }^{*} P<0.05,{ }^{* *} P<0.01,{ }^{* *} P<0.001$. (B) The lower left and lower right panels depict the ANCs and AMCs respectively for each patient in the 3 treatment groups, i.e., placebo, low-frequency CYT107 group, and high-frequency CYT107 group.

even in the patients who received only 3 or 4 doses of CYT107 (Figure 2, B and C). Specifically, at day 42, the absolute lymphocyte count among patients in the low- and high-frequency CYT107 groups were, after baseline adjustment, greater by $1.83 \times 10^{3}(95 \% \mathrm{CI}$ : $0.57,3.18)$ and $1.22 \times 10^{3}$ lymphocytes/ $\mu 1(95 \% \mathrm{CI}$ : $-0.01,2.44$ ), on average relative to the placebo-treated group (combined treatment effect at day 42: $P=0.001$ ) (Supplemental Figure 5). Note that although day 42 was the final time point for which absolute lymphocyte count was quantified, it is likely that the CYT107-induced increase in lymphocytes persisted beyond day 42 . Previous clinical trials of CYT107 have demonstrated that the increase in absolute lymphocyte counts may continue for as long as a year after CYT107 administration is discontinued (31).

\section{CYT107 increased CD4+ and CD8 ${ }^{+}$T cells}

The effect of CYT107 to increase the absolute lymphocyte count was paralleled by its effect to increase $\mathrm{CD}^{+}$and $\mathrm{CD}^{+} \mathrm{T}$ cells, 2 major cell types comprising the absolute lymphocyte count (Figure 3 ). When considered over the entire study period, CYT107 administration had a significant impact on the number of circulating $\mathrm{CD}^{+}$and $\mathrm{CD} 8^{+} \mathrm{T}$ cells (combined treatment effects: $P<0.001$ and $P=0.004$, respectively). At the end of the 28-day treatment regimen, both low- and high-frequency CYT107 dosing schedules caused approximately 4 -fold increases in $\mathrm{CD}^{+} \mathrm{T}$ cells compared with their baseline, pre-CYT107-therapy values. Specifically, after baseline adjustment, at day 29, CD4 ${ }^{+} \mathrm{T}$ cell counts among patients in the lowand high-frequency CYT107-treated groups were greater by $0.32 \times 10^{3}(95 \% \mathrm{CI}$ : $-0.04,0.68)$ and $0.28 \times$ $10^{3}$ lymphocytes $/ \mu 1$ (95\% CI: $\left.-0.07,0.63\right)$, on average relative to the placebo-treated group (combined treatment effect at day 29: $P=0.021$, Supplemental Figure $6 \mathrm{~A}$ ). $\mathrm{CD}^{+} \mathrm{T}$ cell counts among patients in the low-frequency CYT107-treated group were not substantially greater $\left(0.12 \times 10^{3}\right.$ lymphocytes $/ \mu 1 ; 95 \% \mathrm{CI}$ : $-0.66,0.90)$ at day 29 , relative to the placebo-treated group. In the high-frequency CYT107 group at days 29 and $42, \mathrm{CD}^{+} \mathrm{T}$ cell counts were greater by $0.76 \times 10^{3}(95 \% \mathrm{CI}:-0.00,1.52)$ and $1.39 \times 10^{3}$ lymphocytes $/ \mu 1$ (95\% CI: 0.37, 2.31), respectively, relative to placebo (combined treatment effects at days 29 and 42: $P=0.030$ and $P=0.029$, respectively; Supplemental Figure $6 \mathrm{~B}$ ).

\section{CYT107 causes a transient decrease in $\mathrm{CD}^{+}$and $\mathrm{CD} 8^{+} \mathrm{T}$ cells prior to the sustained increase}

Pharmacodynamic studies demonstrate an initial, brief (24-48 hours) decrease in circulating lymphocytes following IL-7 administration. This decrease is coincident with upregulation of lymphocyte adhesion molecules 
Ki67 Expression

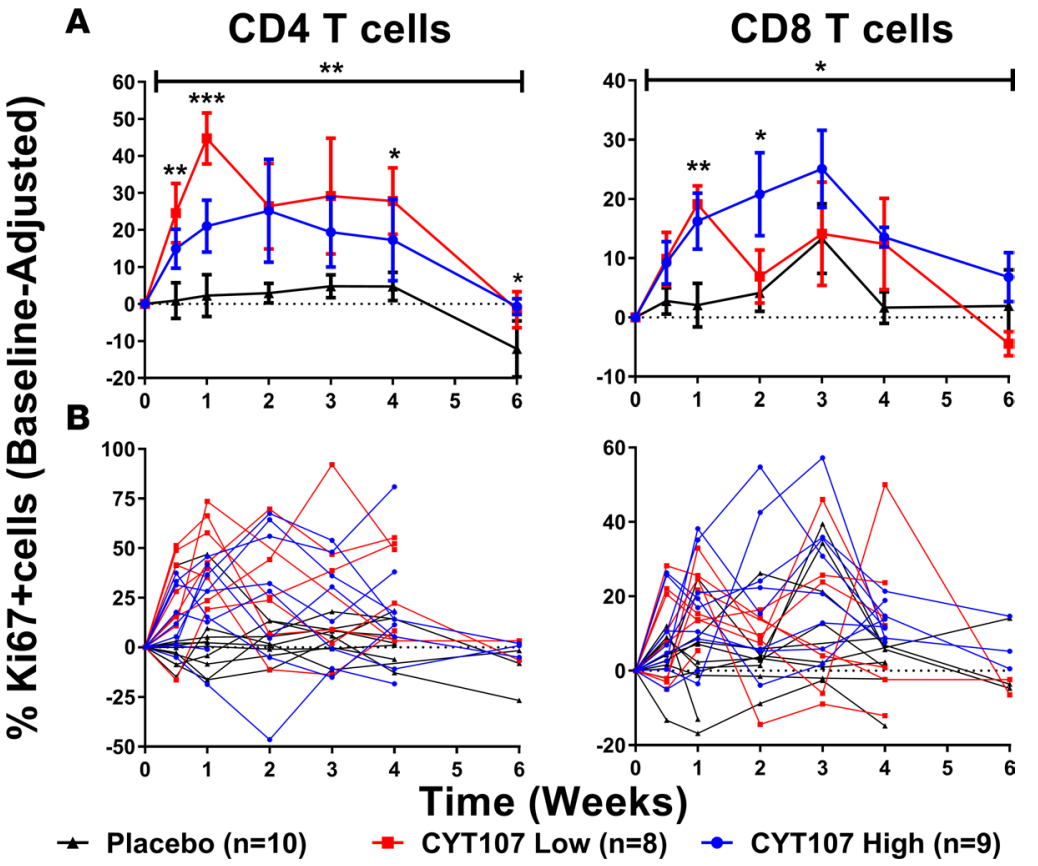

Figure 6. CYT107 increased lymphocyte proliferation. (A) CYT107 increased the percentage of Ki67-positive CD4+ and CD8 ${ }^{+} \mathrm{T}$ cells from approximately $10 \%$ at pre-CYT107 treatment to approximately $46 \%$ and $22 \%$, respectively, on average for both CYT107 treatment groups. In contrast, there was no significant change in the percentage of cells that were Ki67 positive in placebo-treated patients. There was an overall treatment effect on Ki67 in both $\mathrm{CD}^{+}$and $\mathrm{CD} 8^{+} \mathrm{T}$ cells, individually showing an effect of low-dose CYT107 therapy to increase $\mathrm{Ki} 67$ in $\mathrm{CD}^{+} \mathrm{T}$ cells and both regimens to increase Ki67 in CD8 ${ }^{+} \mathrm{T}$ cells as compared with placebo. Additionally, in $\mathrm{CD}^{+} \mathrm{T}$ cells the low-frequency group displayed increased Ki67 at days 4, 8, 22, 29, and 42, while in CD8 ${ }^{+} \mathrm{T}$ cells, the high-frequency group showed increases at days 15 and 29 , and both treatment groups at day 8 as compared with placebo. ${ }^{*} P<0.05,{ }^{*} P<0.01$. Statistical tests were conducted using a Wald-type multiple-degree-of-freedom method. Values reported are mean \pm SEM. $n=10,8$, and 9 for placebo, low-frequency CYT107, and high-frequency CYT107-treated patients, respectively. (B) The effect of CYT107 on lymphocyte proliferation, as determined by flow cytometric quantification of Ki67, is provided for each patient. US study patients had flow cytometric quantification of Ki67 for 6 weeks, while French study patients had flow cytometric quantification out to 4 weeks only. Baseline adjustment reflects the difference between study day values and the predosing values.

and homing of lymphocytes from the circulation and into various tissues such as skin and intestine, and to sites of infection $(39,40)$. In the present study, at day 4 after initiation of CYT107 treatment, CD4 ${ }^{+}$and CD8 ${ }^{+} \mathrm{T}$ cell counts in both low- and high-frequency treatment groups trended in the negative direction being, on average, $31.4 \%$ and $25.4 \%$ lower, respectively, than their day 0 values (Figure 4 ). In contrast, in placebo-treated patients, absolute numbers of $\mathrm{CD}^{+}$and $\mathrm{CD}^{+} \mathrm{T}$ cells increased by $14.4 \%$ and $27.9 \%$, respectively. In comparing CYT107 treatment groups with placebo, the low-frequency CYT107-treated group had a significant reduction in $\mathrm{CD}^{+} \mathrm{T}$ cells by $0.10 \times 10^{3}$ lymphocytes $/ \mu 1$ (95\% CI: $\left.0.03,0.19 ; P=0.009\right)$, on average, at day 4 (Figure 4).

\section{CYT107 effect on absolute neutrophil, monocyte, and B cell counts}

The effect of CYT107 on patients' absolute neutrophil counts (ANCs) and absolute monocyte counts (AMCs) was examined. Early during the course of their sepsis, both CYT107- and placebo-treated patients had increased ANCs compared with normal healthy volunteers (Figure 5 and Supplemental Figure 6, C and D). The ANCs returned to normal values later in the patients' recovery. There was no effect of CYT107 on ANCs through week 2. However, there was an effect of the high- but not low-frequency dosing regimen of CYT107 at week 3 to increase ANCs $(P<0.01)$. In addition to the overall effect, the high-dose group showed increases of $13.16 \times 10^{3}$ cells $/ \mu 1$ (95\% CI: 5.74, 20.58), $7.55 \times 10^{3}$ cells $/ \mu 1$ (95\% CI: $\left.2.38,12.72\right)$, and 5.31 $\times 10^{3}$ cells $/ \mu 1$ (95\% CI: 0.28, 10.33), at days $22(P<0.001), 29(P=0.004)$, and $42(P=0.038)$, respectively (Figure 5 and Supplemental Figure 6D). The statistical difference in ANCs in the CYT107-treated patients versus placebo-treated patients persisted until week 6; however, at that time, the averaged ANCs were within normal limits (shaded area) and quantitative differences between groups were small and of questionable biologic significance. The AMCs were similar for CYT107- and placebo-treated patients and were generally within the normal range for healthy volunteers, (Figure 5 and Supplemental Figure 6C). The effect of CYT107 on the absolute number of B cells was examined in a subset of patients $(n=13)$. There was no apparent effect of CYT107 to increase the absolute number of B cells (Supplemental Figure 7) and these results are in agreement with other clinical studies of CYT107-treated patients (30).

\section{CYT107 increased cell cycling}

To evaluate potential mechanisms for the effect of CYT107 to increase lymphocyte counts, Ki67 was measured in $\mathrm{CD}^{+}$and $\mathrm{CD} 8^{+} \mathrm{T}$ cells by flow cytometry. CYT107-treated patients had a brisk increase in the percentage of Ki67-positive $\mathrm{CD}^{+}$and $\mathrm{CD}^{+} \mathrm{T}$ cells occurring within 4-7 days after starting CYT107 (Figure 6) (combined 


\section{IL-7 Receptor $\alpha$ (CD127) Expression}

A CD4 T cells

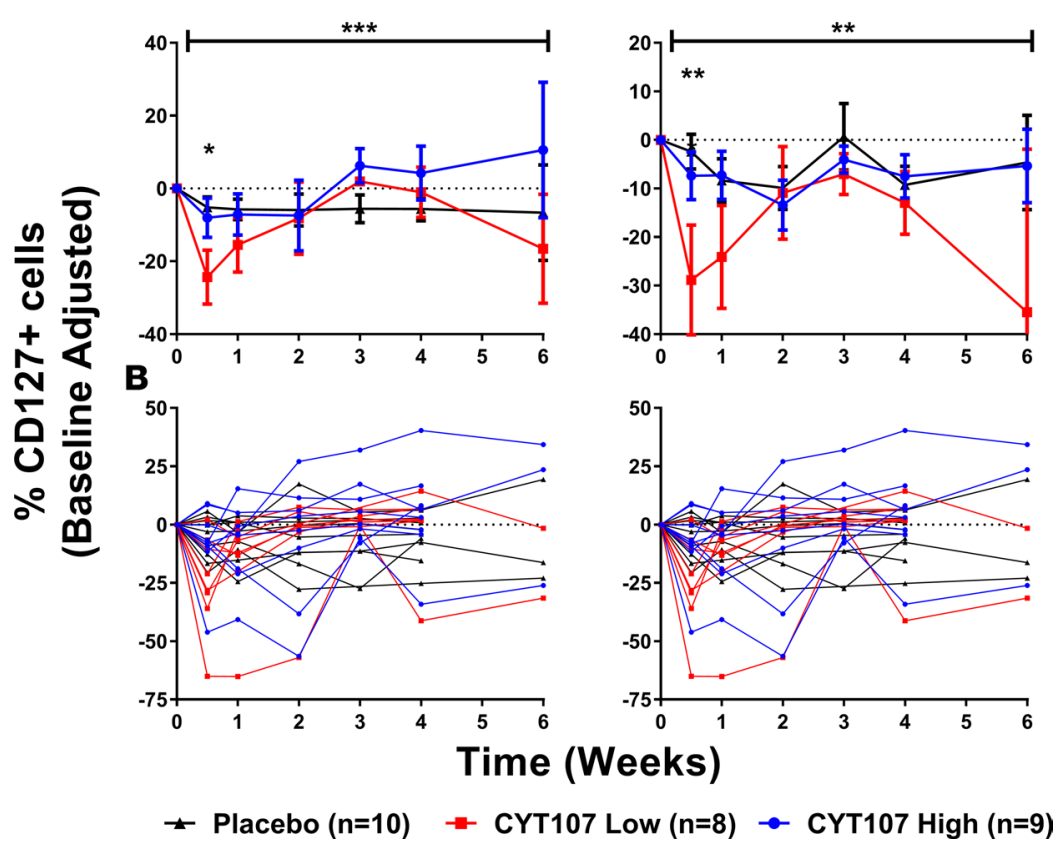

Figure 7. CYT107 decreased IL-7 receptor $\alpha$ (CD127) expression. (A) The IL-7 receptor is composed of a common $\gamma$ chain and the IL-7 receptor $\alpha$ (CD127). CD127 is known to be transiently downregulated after administration of IL-7. Flow cytometry showed that there was a decrease in $\mathrm{CD}^{+}{ }^{+}$and CD8 ${ }^{+} \mathrm{T}$ cell expression of IL-7 receptor $\alpha$ in patients treated with both low- and high-frequency treatment regimens of CYT107 versus placebo at day $4 .{ }^{*} P<0.05$. Statistical tests were conducted using a Wald-type multiple-degree-offreedom method. Values reported are mean \pm SEM for the 3 different treatment groups, i.e., placebo, low-frequency CYT107, and high-frequency CYT107. $n=10,8$, and 9 for placebo, low-frequency CYT107, and high-frequency CYT107treated patients, respectively. (B) The lower left and lower right panels depict the percentages of $\mathrm{CD}^{+}$and $\mathrm{CD} 8^{+} \mathrm{T}$ cells positive for the IL-7 receptor $\alpha$ respectively for each patient in the 3 treatment groups, i.e., placebo, low-frequency CYT107 group, and high-frequency CYT107 group. US study patients had flow cytometric quantification of Ki67 for 6 weeks, while French study patients had flow cytometric quantification out to 4 weeks only. Baseline adjustment reflects the difference between study day values and the predosing values.

treatment effect: $\mathrm{CD} 4^{+} \mathrm{T}, P=0.006 ; \mathrm{CD}^{+} \mathrm{T}, P=0.010$ ). The percentage of Ki67-positive $\mathrm{CD} 4^{+} \mathrm{T}$ cells increased from approximately $10 \%$ at pre-CYT107 treatment to approximately $57.6 \%$ and $37.9 \%$ for low- and high-frequency treatment groups, respectively, versus approximately $15 \%$ for placebo at day 8 . At day 4 , the percentage of Ki67-positive CD4 ${ }^{+} \mathrm{T}$ cells was higher in the low-frequency CYT107-treated group compared with placebo, by $23.6 \%(95 \%$ CI: $6.8,40.5 ; P=0.006)$ on average. Significant differences were also present on days $8(P<0.001)$, $22(P=0.053), 29(P=0.033)$, and $42(P=0.039)$. After adjusting for baseline, there was insufficient evidence of differences in Ki67-positive CD4 ${ }^{+} \mathrm{T}$ cells in the high-frequency CYT107-treated patients versus placebo. CYT107 had a significant impact on Ki67-positive $\mathrm{CD}^{+} \mathrm{T}$ cells (combined treatment effect: $P=0.010$ ). At day 8, Ki67positive $\mathrm{CD}^{+} \mathrm{T}$ cells were greater by $17.1 \%(95 \% \mathrm{CI}: 6.78,27.3 ; P=0.001)$ and $14.2 \%(95 \% \mathrm{CI}: 4.2,24.1 ; P=$ $0.005)$ in the low- and high-frequency CYT107 groups, respectively, on average versus placebo. Significant differences were also present on day $15(P=0.010)$ for the high-frequency treatment group (Figure 6).

\section{CYT107 decreased IL-7 receptor $\alpha$ (CD127) expression}

IL-7 receptor $\alpha(C D 127)$ is a subunit of the IL-7 receptor complex. Studies show that administration of IL-7 to patients results in a rapid and transient downregulation of IL-7 receptor $\alpha(23,32)$. In the present study, there was significant evidence that CYT107 affected CD127 expression on both $\mathrm{CD}^{+} 4$ and CD8 ${ }^{+} \mathrm{T}$ cells (combined treatment effect: $P=0.002$ and $P<0.001$, respectively) (Figure 7). In the low-frequency IL-7 group at day 4, there was a significant decrease in CD127-positive CD4 ${ }^{+}$and $\mathrm{CD} 8^{+} \mathrm{T}$ cells, by $19.1 \%$ (95\% CI: 4.4, 33.9; $P=0.011)$ and $26.04 \%$ (95\% CI: 8.5, 44.4; $P=0.004)$ respectively, versus placebo. Insufficient evidence was found in comparing the high-frequency group versus placebo.

\section{CYT107 increases early lymphocyte activation markers but not PD-1 expression}

We also examined the effect of CYT107 on 2 early T cell activation markers, i.e., CD38 and HLA-DR, and on programmed cell death 1 (PD-1; CD279). There was significant evidence of a combined treatment effect of CYT107 on CD38 in CD4 ${ }^{+} \mathrm{T}$ cells $(P=0.004)$ (Figure 8A). There was no statistically significant effect of CYT107 on CD38 expression in CD8 ${ }^{+} \mathrm{T}$ cells (Supplemental Figure 7A). There was insufficient evidence for a combined treatment effect of CYT107 on HLA-DR expression. Although there was no overall effect by the high-frequency dosing regimen of CYT107 on HLA-DR in CD4 $4^{+} \mathrm{T}$ cells, the effect to increase the percentage of cells expressing HLA-DR did approach statistical significance at days 7 and 22, with an increase from approximately $5 \%$ to an additional $2.20 \%(95 \%$ CI: $-0.19,4.59 ; P=0.07)$ and $2.13 \%(95 \%$ 


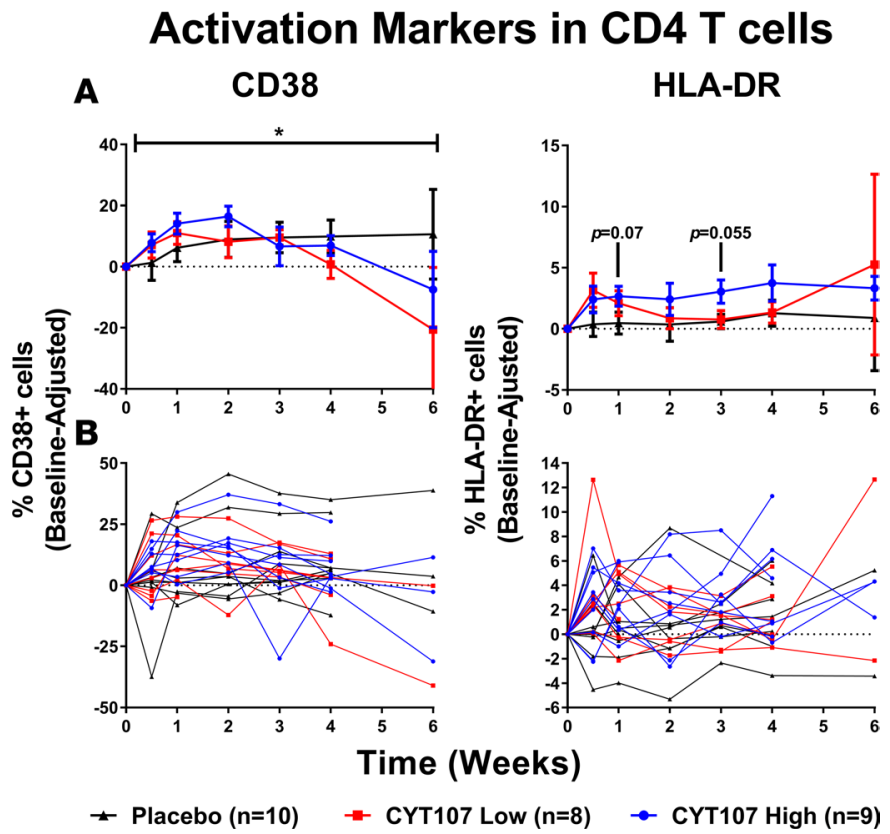

Figure 8. CYT107 increased early activation markers in CD4 ${ }^{+} \mathrm{T}$ cells. (A) The expression of CD38 and HLA-DR increases during T cell activation. The CYT107 treatment effect for CD38, considered for the entire duration of the study, was significant for low-frequency dosing regimen CYT107 (red color) in CD4+ T cells (upper left panel). Additionally, the high-frequency dosing regimen for CYT107 (upper right panel, blue color) showed an increase in HLA-DR expression in CD4 ${ }^{+} T$ cells, which approached significance at days $8(P=0.07)$ and $22(P=0.055)$. ${ }^{*} P<$ 0.05 . Statistical tests were conducted using a Wald-type multipledegree-of-freedom method. Values reported are mean \pm SEM. $n=10,8$, and 9 for placebo, low-frequency CYT107, and high-frequency CYT107treated patients, respectively. (B) The lower left and lower right panels depict the effect of CYT107 on CD38 and HLA-DR expression respectively for each patient in the 3 treatment groups, i.e., placebo, low-frequency CYT107, and high-frequency CYT107. Baseline adjustment reflects the difference between study day values and the predosing values.

CI: $-0.05,4.3 ; P=0.055$ ), respectively (Figure 8B). CYT107 did not increase HLA-DR expression on CD8 ${ }^{+}$ T cells (Supplemental Figure 8A). There was insufficient evidence of an overall effect of either low- or highfrequency regimens of CYT107 on PD-1 expression (Supplemental Figure 8B). The gating strategy used for flow cytometric identification of cell surface markers is illustrated in Supplemental Figures 9 and 10.

\section{Incidence of secondary infections in the treatment groups}

The development of hospital-acquired secondary infections was evaluated by a 3-member panel of experienced intensive care physicians using the Center for Disease Control criteria for healthcare-associated infections. The panel members were blinded to patient identity and treatment group. Four of $10(40 \%)$ of placebo-treated patients versus 5 of 17 (30\%) of CYT107-treated patients developed hospital-acquired infections and this difference was not statistically significant ( $P=0.72$, Fisher's exact test). Sites of secondary infections included ventilator-associated pneumonia, ventilator-associated tracheobronchitis, wound infections, peritonitis, mediastinitis, knee joint infection, and urinary tract infection.

\section{Mortality in CYT107- and placebo-treated patients}

Patients were followed for 120 days after study entry. At the end of the 28-day treatment period, 1 of 10 (10\%) placebo-treated patients and 2 of $17(12 \%)$ of CYT107-treated patients had died $(P=0.855$, Fisher's exact test) (Table 2). At day 120, 2 of 10 (20\%) of placebo-treated patients and 5 of 17 (29\%) of the CYT107treated patients had died and this difference was not statistically significant $(P=0.687$, Fisher's exact test). Causes of death, length of stay in the ICU, and important clinical details that impacted mortality in the patient population are presented in Table 2. Importantly, failure of source control and delayed recognition of ongoing sources of sepsis were contributing factors in mortality in a number of patients. This issue of lack of source control is likely due to the preponderance of surgical ICU patients with sepsis included in this study. One CYT107-treated patient with severe preexisting coronary artery disease and third-degree heart block recovered from his admission for sepsis due to necrotizing fasciitis but died 63 days later of complications from myocardial infarction. One patient in the placebo-treated group died of delayed complications from severe hypoglycemic brain injury when the family decided to change to comfort measures only.

\section{Pharmacokinetics for CYT107}

CYT107 plasma levels were measured at 1, 3, 5, 9, and 24 hours after intramuscular administration at day 1 and day 22 (blue and red curves, respectively; Figure 9). At day 1, the Cmax $443 \mathrm{ng} / \mathrm{ml}$ was observed at 9 hours with an AUC of 7,472.6 pg/ml.h. At day 22, the Cmax $420 \mathrm{ng} / \mathrm{ml}$ was observed at 5 hours with an AUC of $4,358.3 \mathrm{pg} / \mathrm{ml} \cdot \mathrm{h}$. 
Table 2. Mortality for CYT107- and placebo-treated patients

\begin{tabular}{|c|c|c|c|c|c|c|}
\hline Treatment & Age & Sex & Diagnosis & Days ICU & Day expiration & Notes \\
\hline \multirow{4}{*}{ CYT107 $^{A} 29 \%$ (5 out of 17 ) } & 61 & M & $\begin{array}{l}\text { Esophageal leak with mediastinitis } \\
\text { following radiation and esophageal resection }\end{array}$ & 12 & 12 & $\begin{array}{l}\text { Surgical failure (lack of } \\
\text { source control) }\end{array}$ \\
\hline & 65 & M & Mediastinitis following cardiac surgery & 45 & 45 & mesenteric ischemia \\
\hline & 80 & $\mathrm{~F}$ & $\begin{array}{l}\text { Ventilator-associated pneumonia } \\
\text { following motor vehicle accident }\end{array}$ & 63 & 67 & $\begin{array}{l}\text { Prolonged peritonitis due to } \\
\text { unrecognized bile duct injury }\end{array}$ \\
\hline & 71 & M & $\begin{array}{l}\text { Fournier's gangrene complicated by } \\
\text { myocardial infarction and third-degree } \\
\text { heart block }\end{array}$ & 42 & 63 & $\begin{array}{l}\text { Cardiac arrest secondary to } \\
\text { myocardial ischemia }\end{array}$ \\
\hline
\end{tabular}

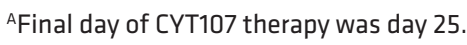

Immunogenicity for detection of antibodies against CYT107

Immunogenicity studies for detection of antibodies against the recombinant human CYT107 employed in this trial were performed at days $0,11,22$, and 60 for all 17 CYT107-treated patients and were negative for antibody detection (see supplemental material).

\section{Discussion}

The present study of CYT107 in patients with sepsis has several potentially unique aspects. First, CYT107's immune-boosting effects stand in marked contrast to the overwhelming majority of previous clinical trials in sepsis that have employed therapies to block inflammation and the host immune response (20, 21, 4145). A second potentially unique aspect is that it represents the first trial to our knowledge that focuses on reversing sepsis-induced defects in adaptive immunity, i.e., $\mathrm{CD} 4^{+}$and $\mathrm{CD} 8^{+} \mathrm{T}$ cells. The few prior immunoadjuvant drug therapy trials that sought to enhance immunity in septic patients employed cytokines and growth factors, e.g., IFN- $\gamma$, G-CSF, and GM-CSF, to increase the number and function of monocytes, macrophages, and neutrophils, which are key cellular components of the innate immune system. In addition to suppressing innate immunity, sepsis significantly impairs adaptive immunity by causing extensive apoptotic death and dysfunction of $\mathrm{CD}^{+}$and $\mathrm{CD}^{+} \mathrm{T}$ cells, the key cellular targets of CYT107 (2-5). In the current study, over $60 \%$ of septic patients had an initial CD4 $4^{+} \mathrm{T}$ cell count of less than 200 cells/ $\mu 1$, one of the criteria used to define AIDS (Figure 4). The potential impact of low CD4 ${ }^{+} \mathrm{T}$ cell counts in sepsis is underscored by reports demonstrating a direct correlation between decreased $\mathrm{CD} 4^{+} \mathrm{T}$ cells and increased incidence of opportunistic infections and death $(12,13)$. Furthermore, numerous investigators have demonstrated a close correlation between low absolute lymphocyte counts and increased mortality in sepsis (5-8). Collectively, these reports, considered together with investigations demonstrating that preventing sepsis-induced lymphocyte depletion improves survival $(25,26,15-18)$, underscore the potential significance of the present findings in which CYT107 caused a 3- to 4-fold increase in absolute lymphocyte counts and $\mathrm{CD}^{+}$and $\mathrm{CD} 8^{+} \mathrm{T}$ cells in patients with septic shock (Figures $2 \mathrm{~B}$ and 3 ).

The profound decrease in circulating lymphocytes in sepsis is mirrored by concomitant decreases in tissue lymphocytes in spleens and other organs in which the number of $\mathrm{CD} 4^{+}$and $\mathrm{CD} 8^{+} \mathrm{T}$ cells is often less than $10 \%-30 \%$ of that present in organs from nonseptic patients $(1,46)$. Although the present study did not address the effect of CYT107 on tissue lymphocytes, numerous studies in animals, including nonhuman primates treated with CYT107 demonstrate that the robust increase in $\mathrm{CD}^{+}$and $\mathrm{CD} 8^{+} \mathrm{T}$ cells present in the blood is accompanied by a significant increase in lymphocytes in spleens and lymph nodes as well (35). Furthermore, HIV-infected patients who had incomplete immune restoration despite receiving antiretroviral therapy and who were treated with CYT107 had markedly increased numbers of $\mathrm{CD}^{+} \mathrm{T}$ cells repopulating their lymphocyte-depleted colons (47). Combined computerized tomography scanning and positron emission tomography (CT-PET) of cancer patients treated with CYT107 showed spleen, mediastinal, and 


\section{CYT107 Pharmacokinetics}

\section{Day 1}

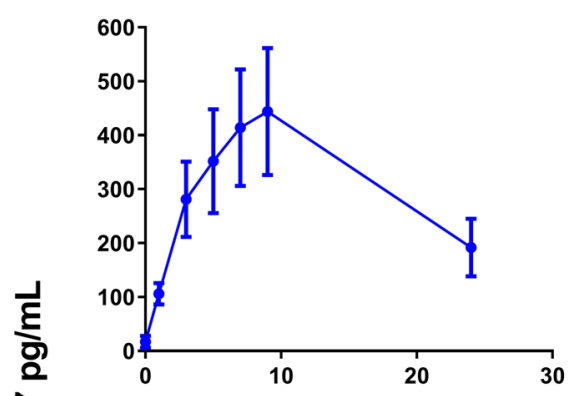

Day 22

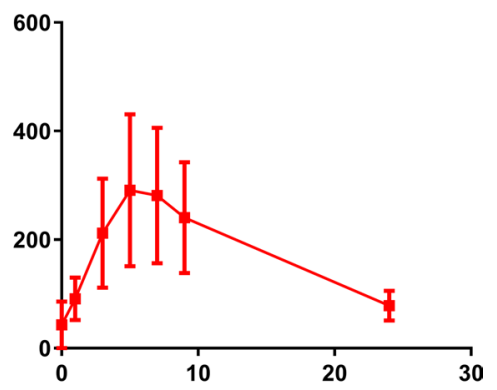

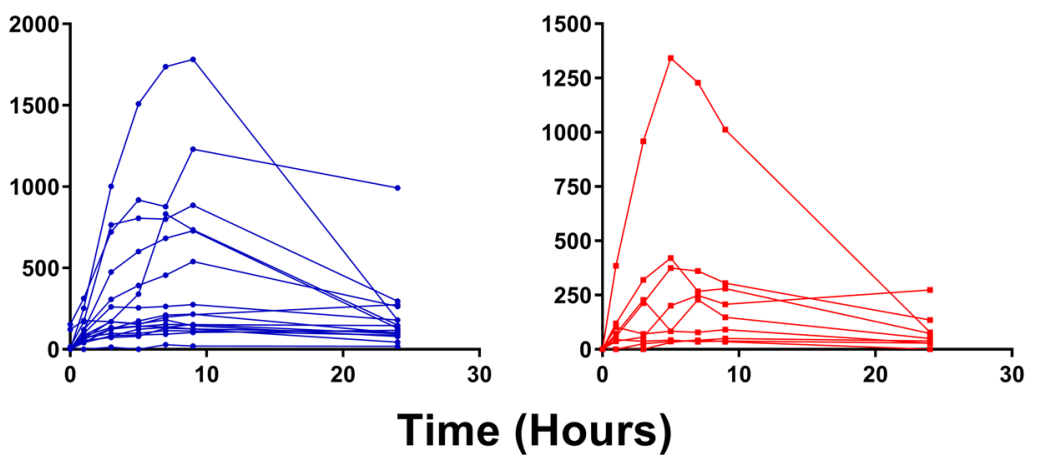

Figure 9. Pharmacokinetics of CYT107. CYT107 (human recombinant CYT107) was measured in patients EDTA-3K plasma at 1, 3, 5, 9, and 24 hours after intramuscular administration. The blue curve shows the kinetics after the first administration at day 1 and the red curve after administration at day 22. Values reported are mean \pm SEM. $n=17$ patients on day 1 and $n=9$ patients on day 22 .

abdominal lymph node enlargement associated with increased cellular metabolic activity in these organs (35). Therefore, the increase in circulating immune effector cells in septic patients treated with CYT107 likely also occurred systemically in the patients' various immune tissues including spleen and lymphoidassociated aggregates in lung and gut.

A seemingly paradoxical effect of CYT107 that has been reported in previous clinical studies of CYT107 is its action to cause an initial drop in circulating lymphocytes. This decrease in circulating lymphocytes lasts $24-72$ hours before rebounding $(39,40)$. Our data showed similar findings, with an early decrease in $\mathrm{CD}^{+} \mathrm{T}$ cell counts and a strong trend toward decreased $\mathrm{CD} 4^{+} \mathrm{T}$ cell counts on day 4 in the CYT107-treated patients (Figure 4). Studies in healthy rhesus macaques established that the transient decrease in circulating $\mathrm{T}$ cells that occurs immediately after the initial dose of CYT107 is due to recruitment of lymphocytes from the bloodstream into various tissues including gut, skin, and lymph nodes $(39,40)$. The presumed mechanism for this trafficking of lymphocytes from the circulation into these tissues is IL-7's effect to upregulate expression of numerous chemokines and adhesion molecules, e.g., CXCR4, CCR7, $\alpha 4 \beta 7$, LFA-1, and VLA-4, on CD4 ${ }^{+}$and CD ${ }^{+} \mathrm{T}$ cells (48-51). This effect of IL-7 to activate lymphocytes to increase expression of adhesion molecules and chemokines is also the likely explanation for the skin rashes that occurred in many CYT107-treated patients. It is possible that CYT107's effect to increase lymphocyte adhesion molecule expression will be advantageous in sepsis by resulting in increased lymphocyte trafficking to sites of infection. Inflammation occurring at sites of infection causes endothelial and parenchymal cells to upregulate the corresponding receptor and/or ligand partners for the adhesion molecules and chemokines expressed on the CD $4^{+}$and $\mathrm{CD} 8^{+}$ $\mathrm{T}$ cells. The overall effect will be migration of immune effector cells to sites of infection and enhanced pathogen killing. In this regard, studies demonstrated that mice with viral hepatitis that were treated with CYT107 had a 3- to 4-fold increase in the number of $\mathrm{CD}^{+}$and $\mathrm{CD} 8^{+} \mathrm{T}$ cells present in the infected liver and an accompanying dramatic improvement in viral clearance compared with control mice (52).

The increase in lymphocytes in CYT107-treated septic patients is likely due to 2 independent mechanisms. First, as is well documented in multiple clinical trials, CYT107 acts to induce proliferation of CD4 ${ }^{+}$ and $\mathrm{CD}^{+} \mathrm{T}$ cells $(31-33,50)$. In the current study, the effect of CYT107 to stimulate lymphocyte proliferation is evidenced by the increase in the cell cycle marker Ki67 in $\mathrm{CD}^{+}$and $\mathrm{CD} 8^{+} \mathrm{T}$ cells of patients treated with CYT107 versus placebo (Figure 5). A second mechanism for the increase in $\mathrm{CD}^{+}$and $\mathrm{CD}^{+} \mathrm{T}$ cells 
in septic patients treated with CYT107 is the potent antiapoptotic effect of IL-7 (25-29). IL-7 increases antiapoptotic Bcl-2 family members and decreases proapoptotic Bcl-2 family members (53). Previously, our laboratory utilized a mouse peritonitis model of sepsis to demonstrate that CYT107 decreased apoptosis of splenic and lymph node naive, central memory, and effector memory $\mathrm{CD}^{+}$and $\mathrm{CD} 8^{+} \mathrm{T}$ cells by approximately $50 \%$ at 24 hours (26). This antiapoptotic effect was associated with an increase in Bcl-2 and a decrease in proapoptotic PUMA. Furthermore, 2 ex vivo studies in septic shock patients' cells showed that IL-7 restored lymphocyte proliferation in response to anti-CD2-CD3-CD28 antibody-coated bead stimulation $(28,29)$. This finding was associated with increased Bcl-2 expression (28) and mTOR pathway reactivation leading to aerobic glycolysis restoration and thus to improved lymphocyte proliferation (29).

Important findings in the present study are data showing that the increase in $\mathrm{CD}^{+}$and $\mathrm{CD} 8^{+} \mathrm{T}$ cells is maintained for the entire duration of the study observation, which was at least 2-4 weeks after cessation of CYT107 therapy (Figures 2B, 2C, and 3). These data are in agreement with numerous clinical studies reporting that the effect of CYT107 to increase lymphocytes persists for several months after the end of CYT107 administration $(32,35)$. This effect of CYT107 to sustain the number of immune effector cells for weeks to months after stopping therapy is likely to be particularly beneficial in sepsis. Many patients survive their initial infection only to develop lethal secondary hospital-acquired infections. These nosocomial infections are a major cause of the approximate $30 \%-40 \%$ additional increase in mortality in patients with sepsis that occurs between days 28 and 90 (54). There was a nonstatistically significant trend toward a $25 \%$ decrease in secondary hospital-acquired infections in patients treated with CYT107 compared with patients receiving placebo.

An interesting finding in the present study is the difference in patient response to low- versus highfrequency CYT107. In some instances, the low-frequency dosing regimen of CYT107 had a more robust effect than the high-frequency dosing regimen (e.g., Figures 6 and 7). We speculate that these differences are due to patient heterogeneity both in extent and type of comorbidities, sites of infection, and infecting pathogens. The fact that some differences were seen within or shortly after the first week (when both the low- and high-frequency CYT107 treatment groups received the same number of doses, i.e., exact same amount of CYT107) suggests that patient heterogeneity and changes in patient clinical status rather than CYT107 was responsible for the observed effect. Similarly, we speculate that the increase in ANCs that was observed in patients treated with the high-frequency CYT107 dose regimen may have been due in part to patient heterogeneity or to changes in patient clinical status rather than an actual effect of CYT107. Neutrophils do not express the IL-7 $\alpha$ receptor and previous clinical trials of CYT107 have not reported increases in neutrophil counts (55). The fact that CYT107 had no effect on the ANCs for the first 2 weeks of therapy and that there were very minor differences in ANCs in CYT107- and placebo-treated patients by week 6 (Figure 5) makes it less likely that CYT107 was having a major effect on the number of circulating neutrophils.

In a very real sense, the immune system is like an orchestra that works optimally when all its diverse members are functioning harmoniously. There is extensive cross talk between the various cellular components of the innate and adaptive immune systems. $\mathrm{CD}^{+}$helper $\mathrm{T}$ cells are particularly essential for proper functioning of host immunity because, as their name indicates, they help to stimulate and instruct the phenotypic response of many other types of innate and adaptive immune effector cells. IL-7 has been called the "maestro of the immune system" because of its effect to activate and induce proliferation of these $\mathrm{CD}^{+}$helper $\mathrm{T}$ cells (56). In this regard, $\mathrm{CD} 4^{+} \mathrm{T}$ cells from patients treated with CYT107 had an increase in the proliferation marker Ki67 and the activation marker CD38 (Figures 6 and 7). As noted previously, the early decrease in circulating $\mathrm{CD}^{+} \mathrm{T}$ cells after initiation of CYT107 therapy is also evidence of $\mathrm{CD}^{+} \mathrm{T}$ cell activation by CYT107. Importantly, CYT107 also increases CD4 ${ }^{+}$and CD8 ${ }^{+} \mathrm{T}$ cell IFN- $\gamma$ production in both animal models of sepsis and in studies of peripheral blood mononuclear cells from patients with sepsis (25-29). IFN- $\gamma$ is a potent activator of monocytes and macrophages, key components of the innate immune system that play an essential role in pathogen killing. Although we did not investigate them, a relatively recently discovered mechanism of action of IL-7 is to increase the number and function of innate lymphoid cells (ILCs) and mucosal-associated invariant T cells (MAITs) (57, 58). ILCs and MAITs, which express the IL-7- $\alpha$ receptor (CD127), are present in high concentrations in lung and gastrointestinal tract. These cells respond rapidly to invading pathogens that enter the host through the pulmonary or gastrointestinal system by producing key cytokines that activate protective antimicrobial immune defenses. The fact that studies demonstrate that IL-7 causes a rapid improvement in survival in 
selected animal models of sepsis within 24-48 hours after its administration may be due to an effect of IL-7 to activate these early responding ILCs and MAITs $(26,59)$.

Unlike some members of the common $\gamma$-chain family of cytokines, CYT107 was well tolerated without evidence of inducing a cytokine storm, exacerbating underlying sepsis-induced inflammation, or worsening existing organ injury (Supplemental Figures 1-3) (23, 24, 30-34). CYT107 has now been reported to be safe in almost 400 adult and pediatric patients with a diversity of disorders including HIV, hepatitis C, cancer, idiopathic lymphopenia, and life-threatening progressive multi-focal leukoencephalopathy (30-37). Furthermore, doses of CYT107 up to 6 times higher than the dose used in this investigation have been administered to oncology patients and reported to be well tolerated (34). One likely explanation for the excellent safety profile of CYT107 is the unique nature of the IL-7 receptor complex, which is partly composed of the IL-7 receptor $\alpha$ subunit (CD127) (23). Administration of CYT107 results in a rapid transitory decreased expression of the IL-7 receptor $\alpha$ chain, thereby rendering the cell less responsive to additional CYT107. Downregulation of IL-7 receptor $\alpha$ presumably prevents overstimulation of $\mathrm{CD} 4^{+}$and $\mathrm{CD} 8^{+} \mathrm{T}$ cells. A statistically significant decrease in IL-7 receptor $\alpha$ expression was observed (Figure 6). The only adverse effect of CYT107 in the present study was a grade 2-3 rash that resulted in early discontinuation of CYT107 dosing in 4 of 17 patients.

The failure of many previous therapeutic trials has generated widespread skepticism in the field toward potential new therapeutic approaches in sepsis (21, 42-45, 57-59). Similar skepticism toward immunotherapy was present in the oncology field prior to the extraordinary advances in cancer treatment that occurred with the discovery of checkpoint inhibitors. Remarkably, the underlying mechanisms responsible for immunosuppression are highly similar in both sepsis and cancer $(60,61)$. Given the common mechanisms of immunosuppression in the 2 disorders, it is not surprising that anti-PD-1, the most widely used and successful immunotherapy in oncology, is also highly effective in restoring $\mathrm{CD}^{+}$and $\mathrm{CD}^{+} \mathrm{T}$ cell function and improving survival in animal models of sepsis (62-66). Anti-PD-1 (nivolumab) is currently in clinical trials in patients with sepsis (NCT02960854). This success of anti-PD-1, a second immunotherapeutic agent whose primary target is blocking a negative regulator of $\mathrm{T}$ cells, provides additional sound theoretical support for the potential efficacy of CYT107 that, like anti-PD-1, acts primarily to restore the number and function of T cells. Both CYT107 and anti-PD-1 represent a transformational therapeutic approach by targeting a hallmark of sepsis, which is massive loss in T cells and the accompanying defect in adaptive immunity in sepsis.

In conclusion, CYT107 represents a promising and potentially novel approach to sepsis and is the first immuno-adjunctive therapy to our knowledge directed at restoring adaptive immunity in this disorder. Although a larger trial of CYT107 in sepsis is needed to confirm findings in the present study, CYT107 appears safe and effective in reversing the profound loss in $\mathrm{CD}^{+}$and $\mathrm{CD} 8^{+}$immune effector cells, a critical pathophysiologic mechanism in sepsis. CYT107 was also effective at increasing T cell activation and potentially assisting in trafficking of $\mathrm{CD}^{+}$and $\mathrm{CD} 8^{+} \mathrm{T}$ cells to sites of infection. The effect of CYT107 to maintain lymphocyte counts continued for several weeks after cessation of drug therapy. Therefore, the beneficial effects of CYT107 on host immunity persist during the time at which surviving patients are highly susceptible to secondary nosocomial infections. The results of the present trial considered in conjunction with CYT107's demonstrable safety record in other critically ill patients and its efficacy in life-threatening viral infections make a compelling case for a definitive trial of CYT107 in this highly lethal disorder.

\section{Methods}

Study design. This study was a prospective, randomized, double-blind, placebo-controlled trial of recombinant human IL-7 (CYT107) in patients with septic shock and severe lymphopenia. Twenty-seven patients at academic sites in France and the United States received CYT107 (in either low- or high-frequency regimens) or placebo for 4 weeks. Primary aims were to determine the safety of CYT107 in sepsis and its ability to reverse lymphopenia. Based on the results of prior trials in patients with HIV or malignancies, a power calculation was performed. A sample size of 10 subjects per trial arm was found to have greater than $85 \%$ power to detect an equal or more extreme treatment effect to reverse lymphopenia.

Study deviations. IL-7 administration was discontinued on 4 patients due to a grade 2-3 rash formation at injection site (see Results section on patient safety). Study enrollment was halted at 27 patients out of a planned enrollment of 30 patients because of requirement for additional drug stability testing that was due at this time. 
Inclusion criteria. Patients had to be between the ages of 18 and 80, have 2 or more criteria for systemic inflammatory response syndrome (SIRS), have a clinically or microbiologically suspected infection (38), a SOFA score of 2 or higher at 48-120 hours after ICU admission, require vasopressor treatment, and an absolute lymphocyte count of 900 cells/ $\mu$ l or lower within 24 hours of a signed informed consent (see supplemental materials).

Exclusion criteria. Patients currently undergoing chemo- or radiotherapy because of cancer, current or a history of hematologic malignancy or lymphoma, cardiopulmonary resuscitation within 4 weeks, history of or current evidence of an autoimmune disorder, organ transplant recipient, HIV/AIDS, hepatitis B or $\mathrm{C}$; receiving corticosteroids at a dose of $300 \mathrm{mg}$ or higher of hydrocortisone/day, receiving biologics that block cytokines were excluded (see supplemental materials).

Thirty-three patients were enrolled into the study but only 27 patients were dosed with drugs (Figure 1). Three patients who were enrolled were not dosed because of marked clinical deterioration with likely impending demise occurring between the time of enrollment and the time of planned administration of drug (CYT107 or placebo). Two patients who consented were not dosed because of rapid clinical improvement within 24-48 hours with resolution of signs of sepsis and SOFA scores less than 2. One patient who was consented was not dosed because of discovery of an exclusion criterion that had been missed earlier.

CYT107 preparation and drug dosing. CYT107, which has been extensively used in other clinical trials, was employed $(30,31)$. Patients were randomized by allocation to a permuted-block randomization (block size of 3 and allocation ratio of 1:1:1, stratified by country, and dosed using 3 different administration protocols: CYT107 high frequency, CYT107 low frequency, and placebo. CYT107 or placebo was administered by intramuscular injection. If the patient's platelet count was below 35,000 per $\mathrm{mm}^{3}$ or the international normalized ratio was 3.5 seconds or higher, CYT107 was administered by subcutaneous injection. All patients were injected twice per week for 4 weeks. All CYT107 patients received $10 \mu \mathrm{g} / \mathrm{kg}$ of CYT107 twice in the first week, followed by: the same dosage, twice per week for the high-frequency regimen; and once per week followed by an injection of placebo $(\mathrm{NaCl} 0.9 \%)$ midweek for the low-frequency regimen. The control, or placebo, group was given placebo $(\mathrm{NaCl} 0.9 \%)$ twice per week.

The statistician, local pharmacist, and the central laboratory in charge of pharmacokinetic (PK)/ immunogenicity analysis were the only unblinded entities.

Blood samples were drawn for flow cytometric analysis prior to the first dose (day 0), following dosing on days $4,8,15$, and 22 , and then on days 29 and 42 . PK samples were drawn on the initial dosing day and on day 22. Immunogenicity samples were collected prior to initial dosing (day 0), and then on days 11, 22, and 60.

Flow cytometry. Flow cytometric analysis was performed as previously described $(28,46)$ - see supplemental materials and methods. Cells stained with cocktails examining only surface markers were fixed in $1 \%$ paraformaldehyde, while cells stained for intracellular markers were first surface stained, permeabilized, and stained for intracellular markers (Ki67). Acquisitions were analyzed with FlowJo v 10.2 (FlowJo, LLC).

Number of $\mathrm{CD}^{+}$and $C D 8^{+} T$ cells. $\mathrm{CD}^{+}$and $\mathrm{CD} 8^{+} \mathrm{T}$ cell numbers were calculated using flow cytometric results. First, the lymphocytes were gated on FSC $\times$ SSC, and then the percentages of $\mathrm{CD} 3^{+} \mathrm{CD} 4^{+}$cells and $\mathrm{CD}^{+} \mathrm{CD}^{+}$cells were determined (Supplemental Figure 5 provides the gating strategy). The frequency of parent percentages was multiplied by absolute lymphocyte count and the product was used as the number of $\mathrm{CD}^{+}$and $\mathrm{CD} 8^{+} \mathrm{T}$ cells, respectively.

Molecules of equivalent soluble fluorochrome (MESF). MESF was calculated by obtaining the population's geometric mean fluorescence intensity (GMFI) and plotting it against values derived from Quantum PE (monocyte HLA-DR, CD279) or Quantum APC (Ki-67, CD127) beads (Bangs Lab) in Microsoft Excel.

Quantification of circulating cytokines IL-6, IL-10, and TNF- $\alpha$ via ELISA. Cytokine kits for TNF- $\alpha$, IL6, and IL-10 were obtained from Biolegend and R\&D Systems - see supplemental materials and methods. Assays were performed exactly as per the manufacturer's instructions and as previously described (46).

$P K$ and immunogenicity analysis. PK and immunogenicity testing were carried out by Eurofins ADME BIOANALYSES.

For PK analysis, CYT107 was measured in EDTA-3K plasma samples using a validated DIACLONE ELISA kit (catalog 851.680.020). The method includes a capture CYT107 antibody and a biotinylated detection antibody. The results obtained during the validation studies are reported in a Eurofins full bioanalytical report.

For immunogenicity testing, anti-CYT107 antibodies were detected in heparin-Li plasma samples. Briefly the ability of plasma samples to bind CYT107 are compared with a positive anti-rh-CYT107 IgG (Preprotech) 
in a preliminary screening test aimed at detecting anti-CYT107 binding antibodies. Positive samples from this screening assay are then spiked with CYT107 to displace and detect the specificity of the binding activity.

Statistics. Comparisons across treatment groups at baseline were performed using the Kruskal-Wallis test and Fisher's exact test. Each outcome variable (e.g., absolute lymphocyte count) was baseline adjusted by subtracting the corresponding baseline value. Baseline-adjusted outcomes were then analyzed using linear mixed-effects regression, adjusting for study day (as a categorical variable), treatment group, and the interaction between study day and treatment group. All figures show values plotted as mean \pm SEM (see supplemental methods for additional details).

Study approval. This double-blind, randomized, placebo-controlled phase IIb trial of CYT107 was conducted at 4 academic sites, with 2 sites in the United States (Vanderbilt University and Washington University ) and 2 sites in France (University Hospital of Limoges and Edouard Herriot Hospital). Following FDA approval of the study, the Institutional Review Boards at Vanderbilt University (IRB 152001) and Washington University (IRB 20161106) approved the protocols. For France, the study protocol was approved by the CPP and ANSM. All patients or their legal representatives gave written informed consent prior to study inclusion.

\section{Author contributions}

BF, SKD, ERS, TD, GM, TR, MM, and RSH conceived and designed the study. RJ, AHW, MSS, JU, AG, TB, MM, GAM, and JB analyzed data and maintained databases. TD, GAM, and JB provided clinical management and study coordination. All authors reviewed the manuscript and discussed results.

\section{Acknowledgments}

This study was supported by funding from Revimmune and by NIH grants GM44118 and GM55194 to R.S. Hotchkiss.

Address correspondence to: Richard S. Hotchkiss, Departments of Anesthesiology, Medicine, and Surgery; 660 South Euclid Avenue, Washington University School of Medicine, St. Louis, Missouri 63110, USA. Phone: 314.362.8552; Email: hotch@wustl.edu.

1. Hotchkiss RS, et al. Apoptotic cell death in patients with sepsis, shock, and multiple organ dysfunction. Crit Care Med. 1999;27(7):1230-1251

2. Hotchkiss RS, Tinsley KW, Karl IE. Role of apoptotic cell death in sepsis. Scand J Infect Dis. 2003;35(9):585-592.

3. Wesche DE, Lomas-Neira JL, Perl M, Chung CS, Ayala A. Leukocyte apoptosis and its significance in sepsis and shock. J Leukoc Biol. 2005;78(2):325-337.

4. Hotchkiss RS, et al. Sepsis-induced apoptosis causes progressive profound depletion of B and CD4 ${ }^{+} \mathrm{T}$ lymphocytes in humans. J Immunol. 2001;166(11):6952-6963.

5. Drewry AM, Samra N, Skrupky LP, Fuller BM, Compton SM, Hotchkiss RS. Persistent lymphopenia after diagnosis of sepsis predicts mortality. Shock. 2014;42(5):383-391.

6. Inoue $\mathrm{S}$, et al. Reduction of immunocompetent $\mathrm{T}$ cells followed by prolonged lymphopenia in severe sepsis in the elderly. Crit Care Med. 2013;41(3):810-819.

7. de Jager CP, van Wijk PT, Mathoera RB, de Jongh-Leuvenink J, van der Poll T, Wever PC. Lymphocytopenia and neutrophillymphocyte count ratio predict bacteremia better than conventional infection markers in an emergency care unit. Crit Care. 2010;14(5):R192.

8. Heffernan DS, Monaghan SF, Thakkar RK, Machan JT, Cioffi WG, Ayala A. Failure to normalize lymphopenia following trauma is associated with increased mortality, independent of the leukocytosis pattern. Crit Care. 2012;16(1):R12.

9. Hotchkiss RS, Osmon SB, Chang KC, Wagner TH, Coopersmith CM, Karl IE. Accelerated lymphocyte death in sepsis occurs by both the death receptor and mitochondrial pathways. J Immunol. 2005;174(8):5110-5118.

10. Venet F, et al. Early assessment of leukocyte alterations at diagnosis of septic shock. Shock. 2010;34(4):358-363.

11. Le Tulzo Y, et al. Early circulating lymphocyte apoptosis in human septic shock is associated with poor outcome. Shock. 2002;18(6):487-494.

12. Small TN, et al. Comparison of immune reconstitution after unrelated and related T-cell-depleted bone marrow transplantation: effect of patient age and donor leukocyte infusions. Blood. 1999;93(2):467-480.

13. Savani BN, et al. Absolute lymphocyte count on day 30 is a surrogate for robust hematopoietic recovery and strongly predicts outcome after T cell-depleted allogeneic stem cell transplantation. Biol Blood Marrow Transplant. 2007;13(10):1216-1223.

14. Hotchkiss RS, et al. Overexpression of Bcl-2 in transgenic mice decreases apoptosis and improves survival in sepsis. J Immunol. 1999;162(7):4148-4156.

15. Chang KC, et al. Multiple triggers of cell death in sepsis: death receptor and mitochondrial-mediated apoptosis. FASEB J. 2007;21(3):708-719.

16. Iwata A, et al. Over-expression of Bcl-2 provides protection in septic mice by a trans effect. J Immunol. 2003;171(6):3136-3141. 
17. Oberholzer C, et al. Targeted adenovirus-induced expression of IL-10 decreases thymic apoptosis and improves survival in murine sepsis. Proc Natl Acad Sci USA. 2001;98(20):11503-11508.

18. Chung CS, Song GY, Lomas J, Simms HH, Chaudry IH, Ayala A. Inhibition of Fas/Fas ligand signaling improves septic survival: differential effects on macrophage apoptotic and functional capacity. J Leukoc Biol. 2003;74(3):344-351.

19. Wesche-Soldato DE, Swan RZ, Chung CS, Ayala A. The apoptotic pathway as a therapeutic target in sepsis. Curr Drug Targets. 2007;8(4):493-500.

20. Rittirsch D, Flierl MA, Ward PA. Harmful molecular mechanisms in sepsis. Nat Rev Immunol. 2008;8(10):776-787.

21. Delano MJ, Ward PA. Sepsis-induced immune dysfunction: can immune therapies reduce mortality? J Clin Invest. 2016;126(1):23-31.

22. Hofmeister R, Khaled AR, Benbernou N, Rajnavolgyi E, Muegge K, Durum SK. Interleukin-7: physiological roles and mechanisms of action. Cytokine Growth Factor Rev. 1999;10(1):41-60.

23. Mazzucchelli R, Durum SK. Interleukin-7 receptor expression: intelligent design. Nat Rev Immunol. 2007;7(2):144-154.

24. Mackall CL, Fry TJ, Gress RE. Harnessing the biology of IL-7 for therapeutic application. Nat Rev Immunol. 2011;11(5):330-342.

25. Unsinger J, et al. Interleukin-7 ameliorates immune dysfunction and improves survival in a 2-hit model of fungal sepsis. $J$ Infect Dis. 2012;206(4):606-616.

26. Unsinger J, et al. IL-7 promotes T cell viability, trafficking, and functionality and improves survival in sepsis. J Immunol. 2010;184(7):3768-3779.

27. Kasten KR, et al. Interleukin-7 (IL-7) treatment accelerates neutrophil recruitment through gamma delta T-cell IL-17 production in a murine model of sepsis. Infect Immun. 2010;78(11):4714-4722.

28. Venet F, et al. IL-7 restores lymphocyte functions in septic patients. J Immunol. 2012;189(10):5073-5081.

29. Venet F, et al. IL-7 restores T lymphocyte immunometabolic failure in septic shock patients through mTOR activation. $J$ Immunol. 2017;199(5):1606-1615.

30. Perales MA, et al. Recombinant human interleukin-7 (CYT107) promotes T-cell recovery after allogeneic stem cell transplantation. Blood. 2012;120(24):4882-4891.

31. Lévy Y, et al. Effects of recombinant human interleukin 7 on T-cell recovery and thymic output in HIV-infected patients receiving antiretroviral therapy: results of a phase I/IIa randomized, placebo-controlled, multicenter study. Clin Infect Dis. 2012;55(2):291-300.

32. Sereti I, et al. IL-7 administration drives T cell-cycle entry and expansion in HIV-1 infection. Blood. 2009;113(25):6304-6314

33. Sheikh V, et al. Administration of interleukin-7 increases CD4 T cells in idiopathic CD4 lymphocytopenia. Blood. 2016;127(8):977-988.

34. Rosenberg SA, et al. IL-7 administration to humans leads to expansion of CD8 ${ }^{+}$and $C D 4^{+}$cells but a relative decrease of CD4 ${ }^{+}$ T-regulatory cells. J Immunother. 2006;29(3):313-319.

35. Sportès C, et al. Administration of rhIL-7 in humans increases in vivo TCR repertoire diversity by preferential expansion of naive T cell subsets. J Exp Med. 2008;205(7):1701-1714.

36. Patel A, Patel J, Ikwuagwu J. A case of progressive multifocal leukoencephalopathy and idiopathic CD4 ${ }^{+}$lymphocytopenia. J Antimicrob Chemother. 2010;65(12):2697-2698.

37. Alstadhaug KB, et al. Treatment of progressive multifocal leukoencephalopathy with interleukin 7. JAMA Neurol. 2014;71(8):1030-1035.

38. Bone RC, et al. Definitions for sepsis and organ failure and guidelines for the use of innovative therapies in sepsis. The ACCP/ SCCM Consensus Conference Committee. American College of Chest Physicians/Society of Critical Care Medicine. Chest. 1992;101(6):1644-1655.

39. Beq S, et al. Injection of glycosylated recombinant simian IL-7 provokes rapid and massive T-cell homing in rhesus macaques. Blood. 2009;114(4):816-825.

40. Sereti I. Where have all the T cells gone? Blood. 2009;114(4):751-752.

41. van der Poll T, van de Veerdonk FL, Scicluna BP, Netea MG. The immunopathology of sepsis and potential therapeutic targets. Nat Rev Immunol. 2017;17(7):407-420.

42. Cohen J, Opal S, Calandra T. Sepsis studies need new direction. Lancet Infect Dis. 2012;12(7):503-505.

43. Hotchkiss RS, Karl IE. The pathophysiology and treatment of sepsis. N Engl J Med. 2003;348(2):138-150.

44. Angus DC. The search for effective therapy for sepsis: back to the drawing board? JAMA. 2011;306(23):2614-2615.

45. Cohen J, et al. Sepsis: a roadmap for future research. Lancet Infect Dis. 2015;15(5):581-614.

46. Boomer JS, et al. Immunosuppression in patients who die of sepsis and multiple organ failure. JAMA. 2011;306(23):2594-2605.

47. Sereti I, et al. Decreases in colonic and systemic inflammation in chronic HIV infection after IL-7 administration. PLoS Pathog. 2014;10(1):e1003890.

48. Cimbro R, et al. IL-7 induces expression and activation of integrin $\alpha 4 \beta 7$ promoting naive T-cell homing to the intestinal mucosa. Blood. 2012;120(13):2610-2619.

49. Kittipatarin C, Li W, Durum SK, Khaled AR. Cdc25A-driven proliferation regulates CD62L levels and lymphocyte movement in response to interleukin-7. Exp Hematol. 2010;38(12):1143-1156.

50. Li WQ, Jiang Q, Aleem E, Kaldis P, Khaled AR, Durum SK. IL-7 promotes T cell proliferation through destabilization of p27Kip1. J Exp Med. 2006;203(3):573-582.

51. Kitazawa H, et al. IL-7 activates alpha4beta1 integrin in murine thymocytes. J Immunol. 1997;159(5):2259-2264.

52. Pellegrini M, et al. IL-7 engages multiple mechanisms to overcome chronic viral infection and limit organ pathology. Cell. 2011;144(4):601-613.

53. Khaled AR, Durum SK. Death and Baxes: mechanisms of lymphotrophic cytokines. Immunol Rev. 2003;193:48-57.

54. Opal SM, et al. Effect of eritoran, an antagonist of MD2-TLR4, on mortality in patients with severe sepsis: the ACCESS randomized trial. JAMA. 2013;309(11):1154-1162.

55. Girard D, Beaulieu AD. Absence of the IL-7 receptor component CDw127 indicates that gamma(c) expression alone is insufficient for IL-7 to modulate human neutrophil responses. Clin Immunol Immunopathol. 1997;83(3):264-271.

56. Sprent J, Surh CD. Interleukin 7, maestro of the immune system. Semin Immunol. 2012;24(3):149-150. 
57. Sonnenberg GF, Artis D. Innate lymphoid cells in the initiation, regulation and resolution of inflammation. Nat Med. 2015;21(7):698-708.

58. Leeansyah E, et al. Arming of MAIT cell cytolytic antimicrobial activity is induced by IL-7 and defective in HIV-1 infection. PLoS Pathog. 2015;11(8):e1005072.

59. Shindo Y, et al. Interleukin 7 immunotherapy improves host immunity and survival in a two-hit model of Pseudomonas aeruginosa pneumonia. J Leukoc Biol. 2017;101(2):543-554.

60. Angus DC, van der Poll T. Severe sepsis and septic shock. NEngl J Med. 2013;369(9):840-851.

61. Vincent JL. Emerging therapies for the treatment of sepsis. Curr Opin Anaesthesiol. 2015;28(4):411-416.

62. Delano MJ, Ward PA. The immune system's role in sepsis progression, resolution, and long-term outcome. Immunol Rev. 2016;274(1):330-353.

63. Cheever MA. Twelve immunotherapy drugs that could cure cancers. Immunol Rev. 2008;222:357-368.

64. Hotchkiss RS, Moldawer LL. Parallels between cancer and infectious disease. N Engl J Med. 2014;371(4):380-383

65. Huang X, et al. PD-1 expression by macrophages plays a pathologic role in altering microbial clearance and the innate inflammatory response to sepsis. Proc Natl Acad Sci USA. 2009;106(15):6303-6308.

66. Brahmamdam P, Inoue S, Unsinger J, Chang KC, McDunn JE, Hotchkiss RS. Delayed administration of anti-PD-1 antibody reverses immune dysfunction and improves survival during sepsis. J Leukoc Biol. 2010;88(2):233-240. 